\title{
Asymptotics of greedy energy sequences on the unit circle and the sphere
}

\author{
Abey López-García* Ryan E. McCleary ${ }^{\dagger}$
}

August 12, 2021

\begin{abstract}
For a parameter $\lambda>0$, we investigate greedy $\lambda$-energy sequences $\left(a_{n}\right)_{n=0}^{\infty}$ on the unit sphere $S^{d} \subset \mathbb{R}^{d+1}, d \geq 1$, satisfying the defining property that each $a_{n}, n \geq 1$, is a point where the potential $\sum_{k=0}^{n}-1\left|x-a_{k}\right|^{\lambda}$ attains its maximum value on $S^{d}$. We show that these sequences satisfy the symmetry property $a_{2 k+1}=-a_{2 k}$ for every $k \geq 0$. The asymptotic distribution of the sequence undergoes a sharp transition at the value $\lambda=2$, from uniform distribution $(\lambda<2)$ to concentration on two antipodal points $(\lambda>2)$. We investigate firstorder and second-order asymptotics of the $\lambda$-energy of the first $N$ points of the sequence, as well as the asymptotic behavior of the extremal values $\sum_{k=0}^{n-1}\left|a_{n}-a_{k}\right|^{\lambda}$. The second-order asymptotics is analyzed on the unit circle. It is shown that this asymptotic behavior differs significantly from that of $N$ equally spaced points on the unit circle, and a transition in the behavior takes place at $\lambda=1$.
\end{abstract}

Keywords: Greedy $\lambda$-energy sequence, Leja sequence, maximal distribution, potential, binary representation, Riemann zeta function.

MSC 2020: Primary 31C20, 31B15; Secondary 11M06.

\section{Introduction}

Let $S^{d}=\left\{x \in \mathbb{R}^{d+1}:|x|=1\right\}$ be the unit sphere of dimension $d \geq 1$. In this paper, $d$ will always refer to the dimension of the sphere we are considering, and so it is always a positive integer. Let $\lambda>0$, and let $\omega_{N}=\left(y_{k}\right)_{k=0}^{N-1}$ be a configuration of $N \geq 2$ points on $S^{d}$, not necessarily distinct. We define

$$
H_{\lambda}\left(\omega_{N}\right):=\sum_{0 \leq i \neq j \leq N-1}\left|y_{i}-y_{j}\right|^{\lambda}=2 \sum_{0 \leq i<j \leq N-1}\left|y_{i}-y_{j}\right|^{\lambda},
$$

where $|\cdot|$ indicates the Euclidean norm.

The goal of this paper is the investigation of sequences $\left(a_{n}\right)_{n=0}^{\infty} \subset S^{d}$ that satisfy the following condition:

$$
\sum_{k=0}^{n-1}\left|a_{n}-a_{k}\right|^{\lambda}=\max _{x \in S^{d}} \sum_{k=0}^{n-1}\left|x-a_{k}\right|^{\lambda}, \quad \text { for all } n \geq 1 .
$$

One can view (1.2) as a recursive algorithm that generates the entire sequence $\left(a_{n}\right)_{n=0}^{\infty}$, starting from an initial input $a_{0}$. Note that for a given $n \geq 2$, the choice of a point $a_{n} \in S^{d}$ that satisfies

\footnotetext{
*Department of Mathematics, University of Central Florida, 4393 Andromeda Loop North, Orlando, FL 32816, USA. email: abey.lopez-garcia@ucf.edu.

${ }^{\dagger}$ Department of Mathematics, University of Central Florida, 4393 Andromeda Loop North, Orlando, FL 32816, USA. email: remccleary@knights.ucf.edu.
} 
(1.2) need not be unique. When studying these sequences in the special framework of the unit circle $S^{1}$, we will assume for convenience that the first point in the sequence is

$$
a_{0}=1 \text {. }
$$

Similarly defined sequences, satisfying a condition as in (1.2) but instead minimizing the Riesz potential $\sum_{k=0}^{n-1}\left|x-a_{k}\right|^{-s}, s>0$, or logarithmic potential $\sum_{k=0}^{n-1} \log \frac{1}{\left|x-a_{k}\right|}$, include the wellknown Leja (also called Leja-Górski) sequences. These sequences were first studied from the point of view of their energy asymptotics and distribution by Edrei [6] ${ }^{\S}$, Leja [10], Górski [7], and Siciak [17. Recently, potential-theoretic properties of these extremal sequences were investigated in [5, 8, 9, 11, 12, 13, 14, 15, 16. In [12, more general greedy energy sequences associated with general kernels were defined and studied. By analogy, we will also refer to the sequences investigated in the present work as greedy $\lambda$-energy sequences.

For a greedy $\lambda$-energy sequence $\left(a_{n}\right)_{n=0}^{\infty} \subset S^{d}$ satisfying (1.2), we will use the notation

$$
\alpha_{N, \lambda}:=\left(a_{0}, \ldots, a_{N-1}\right), \quad N \geq 1 .
$$

We call $\alpha_{N, \lambda}$ the $N$-th section of the sequence. If the value of $\lambda$ is clear from the context, we may simply write $\alpha_{N}$, or $H\left(\alpha_{N}\right)$ in reference to the energy (1.1) of $\alpha_{N}$.

We will denote by $\sigma_{N, \lambda}$ the normalized counting measure

$$
\sigma_{N, \lambda}:=\frac{1}{N} \sum_{k=0}^{N-1} \delta_{a_{k}}
$$

associated with the $N$ th section $\alpha_{N, \lambda}$, where $\delta_{a}$ is the Dirac unit measure at $a$. If $\mu$ and $\left(\mu_{n}\right)_{n \in \mathbb{N}}$ are probability measures on $S^{d}$, then

$$
\mu_{n} \stackrel{*}{\longrightarrow} \mu
$$

means that for every continuous function $f: S^{d} \longrightarrow \mathbb{R}$, we have

$$
\lim _{n \rightarrow \infty} \int f d \mu_{n}=\int f d \mu .
$$

In this paper, $\mathcal{M}_{d}$ denotes the space of all probability distributions on $S^{d}$, and $\sigma_{d} \in \mathcal{M}_{d}$ denotes the normalized uniform measure on $S^{d}$. Given $\mu \in \mathcal{M}_{d}$, we define its $\lambda$-energy

$$
I_{\lambda}(\mu):=\iint_{S^{d} \times S^{d}}|x-y|^{\lambda} d \mu(x) d \mu(y), \quad \lambda>0 .
$$

For a given $\lambda>0$, a distribution $\sigma \in \mathcal{M}_{d}$ is called maximal if

$$
I_{\lambda}(\sigma)=\sup _{\mu \in \mathcal{M}_{d}} I_{\lambda}(\mu)
$$

The existence of maximal distributions follows from a standard convergence argument in potential theory. Maximal distributions were described by Björck in [2]. We cite his result.

Theorem 1.1 (Björck [2]). For $0<\lambda<2$, the measure $\sigma_{d}$ is the unique maximal distribution in $\mathcal{M}_{d}$. For $\lambda=2$, a distribution $\sigma \in \mathcal{M}_{d}$ is maximal if and only if its center of mass is at the origin, i.e., it satisfies (4.7). For $\lambda>2$, a distribution $\sigma \in \mathcal{M}_{d}$ is maximal if and only if it is of the form $\sigma=\frac{1}{2}\left(\delta_{a}+\delta_{-a}\right)$ for some $a \in S^{d}$.

\footnotetext{
§In [6], Leja sequences on a compact set $E \subset \mathbb{C}$ are introduced in page 78 . Edrei then shows that the normalized Vandermonde determinant $\left|V\left(a_{0}, \ldots, a_{n-1}\right)\right|^{2 / n^{2}}$ approaches the transfinite diameter of $E$.
} 
We remark that for $0<\lambda<2$, we have

$$
I_{\lambda}\left(\sigma_{d}\right)=\frac{\Gamma\left(\frac{d+1}{2}\right) \Gamma(d+\lambda)}{\Gamma\left(\frac{d+\lambda+1}{2}\right) \Gamma\left(d+\frac{\lambda}{2}\right)}=\frac{2^{d+\lambda-1}}{\sqrt{\pi}} \frac{\Gamma\left(\frac{d+1}{2}\right) \Gamma\left(\frac{d+\lambda}{2}\right)}{\Gamma\left(d+\frac{\lambda}{2}\right)},
$$

see e.g. [3, Proposition 4.6.4], and for $\lambda \geq 2$, and any corresponding maximal distribution $\sigma$,

$$
I_{\lambda}(\sigma)=2^{\lambda-1}
$$

see [2, Theorems 6 and 7].

In this paper, $U_{n}(x)=U_{n}^{\lambda}(x)$ will denote the discrete potential

$$
U_{n}(x):=\sum_{k=0}^{n-1}\left|x-a_{k}\right|^{\lambda}, \quad n \geq 1 .
$$

Since the value of $\lambda$ will be clear from the context, we will omit the superscript $\lambda$ when indicating $U_{n}$.

Our main goal in this work is the asymptotic analysis of the sequences $H_{\lambda}\left(\alpha_{N, \lambda}\right), U_{n}\left(a_{n}\right)$, and $\sigma_{N, \lambda}$. We first show that for any values of $d \geq 1$ and $\lambda>0$, greedy $\lambda$-energy sequences $\left(a_{n}\right)_{n=0}^{\infty} \subset S^{d}$ satisfy the symmetry property

$$
a_{2 k+1}=-a_{2 k}, \quad \text { for all } \quad k \geq 0,
$$

see Theorem 2.1. This property allows us to compute half the points of a greedy sequence automatically and concentrate the computational effort on obtaining the points $a_{2 k}$ with even index. In the range $0<\lambda<2$, greedy sequences are uniformly distributed on $S^{d}$, see (3.3), whereas in the case $\lambda>2$, we have $\left\{a_{2 k}, a_{2 k+1}\right\}=\left\{a_{0}, a_{1}\right\}$ for all $k \geq 0$, so the sequence is restricted to its first two points, see Theorem [5.2. In the intermediate case $\lambda=2$, a sequence $\left(a_{n}\right)$ is a greedy 2-energy sequence if and only if it satisfies (1.8), and so in this case it is possible that $\left(\sigma_{N, 2}\right)$ diverges, see Theorem 4.1 and Remark 4.4

In the range $0<\lambda<2$, the first-order asymptotic behavior of the sequences $\left(H_{\lambda}\left(\alpha_{N, \lambda}\right)\right)$ and $\left(U_{n}\left(a_{n}\right)\right)$ is described by formulas (3.2) and (3.12). These formulas immediately suggest the study of the sequences $\left(H_{\lambda}\left(\alpha_{N, \lambda}\right)-N^{2} I_{\lambda}\left(\sigma_{d}\right)\right)$ and $\left(U_{n}\left(a_{n}\right)-n I_{\lambda}\left(\sigma_{d}\right)\right)$, or second-order asymptotics, which we develop in detail in dimension $d=1$. The asymptotic analysis of the sequence $\left(H_{\lambda}\left(\alpha_{N, \lambda}\right)-N^{2} I_{\lambda}\left(\sigma_{1}\right)\right)$ needs to be divided in three regimes; $0<\lambda<1, \lambda=1$, $1<\lambda<2$, as we have different orders of growth $H_{\lambda}\left(\alpha_{N, \lambda}\right)-N^{2} I_{\lambda}\left(\sigma_{1}\right)=O\left(\kappa_{\lambda}(N)\right), N \rightarrow \infty$, where

$$
\kappa_{\lambda}(N)= \begin{cases}N^{1-\lambda} & 0<\lambda<1 \\ \log N & \lambda=1 \\ 1 & 1<\lambda<2 .\end{cases}
$$

It turns out that the bounded sequences $\left(\left(H_{\lambda}\left(\alpha_{N, \lambda}\right)-N^{2} I_{\lambda}\left(\sigma_{1}\right)\right) / \kappa_{\lambda}(N)\right)$ are also divergent, see Theorems 3.16, 3.17, and 3.18.

Let $\mathcal{L}_{\lambda}(N)$ denote the $\lambda$-energy of the configuration formed by the $N$ th roots of unity on the unit circle. ${ }^{\S}$ If we compare, in the range $0<\lambda<2$ and on the unit circle, the asymptotics of $H_{\lambda}\left(\alpha_{N, \lambda}\right)$ and $\mathcal{L}_{\lambda}(N)$, we see that they coincide in first-order asymptotics, but they differ significantly at the level of second-order asymptotics. Indeed, we know by [4, Theorem 1.1] that $\mathcal{L}_{\lambda}(N)-I_{\lambda}\left(\sigma_{1}\right) N^{2}=O\left(N^{1-\lambda}\right)$ for all $\lambda \in(0,2)$, and the sequence $\left(\mathcal{L}_{\lambda}(N)-I_{\lambda}\left(\sigma_{1}\right) N^{2}\right) / N^{1-\lambda}$ converges.

\footnotetext{
${ }^{\S}$ In the range $0<\lambda<2, \mathcal{L}_{\lambda}(N)$ is the largest value for the $\lambda$-energy of an $N$-point configuration on $S^{1}$.
} 
In the range $0<\lambda<2$, we show that the sequence $\left(U_{n}\left(a_{n}\right)-n I_{\lambda}\left(\sigma_{1}\right)\right)$ is bounded and divergent, and we have $0<U_{n}\left(a_{n}\right)-n I_{\lambda}\left(\sigma_{1}\right)<I_{\lambda}\left(\sigma_{1}\right)$ for all $n \geq 1$, where the bounds are sharp, see Theorem 3.11 .

We illustrate graphically the sequences $\left(U_{n}\left(a_{n}\right)-n I_{\lambda}\left(\sigma_{1}\right)\right)$ (see Figs. 1, 2) and $\left(\left(H_{\lambda}\left(\alpha_{N, \lambda}\right)-\right.\right.$ $\left.N^{2} I_{\lambda}\left(\sigma_{1}\right)\right) / \kappa_{\lambda}(N)$ ) (see Figs. 4, 5, 6). These plots show a rather regular and rich behavior of these sequences, which was not anticipated by the authors.

The organization of this paper is simple. In Section 2 we prove the symmetry property. In the rest of the sections, we organize the results obtained in the different ranges for $\lambda$, namely $0<\lambda<2, \lambda=2$, and $\lambda>2$.

\section{Symmetry property}

Theorem 2.1. Let $\lambda>0$ and $d \geq 1$ be arbitrary, and let $\left(a_{n}\right)_{n=0}^{\infty} \subset S^{d}$ be a greedy $\lambda$-energy sequence. For any odd index $n=2 k+1, k \geq 0$, the function $U_{n}(x)=\sum_{j=0}^{n-1}\left|x-a_{j}\right|^{\lambda}$ attains its maximum value at a unique point on $S^{d}$, which is $-a_{n-1}=-a_{2 k}$, i.e., we have

$$
a_{2 k+1}=-a_{2 k}, \quad \text { for all } \quad k \geq 0 .
$$

For any even index $n=2 k \geq 2$, we have

$$
U_{2 k}(x)=U_{2 k}(-x), \quad \text { for all } x \in S^{d} .
$$

Proof. First, it is obvious that $a_{1}=-a_{0}$. Now assume that $a_{2 k+1}=-a_{2 k}$ for all $0 \leq k \leq l$. Let

$$
U(x):=\sum_{k=0}^{l}\left(\left|x-a_{2 k}\right|^{\lambda}+\left|x-a_{2 k+1}\right|^{\lambda}\right), \quad x \in S^{d} .
$$

By assumption, this can be rewritten as

$$
U(x)=\sum_{k=0}^{l}\left(\left|x-a_{2 k}\right|^{\lambda}+\left|x+a_{2 k}\right|^{\lambda}\right),
$$

hence $U(x)=U(-x)$ for all $x \in S^{d}$. By definition, $a_{2 l+2}$ must maximize $U$. We will show now that the choice of $a_{2 l+3}$ is unique and that $a_{2 l+3}=-a_{2 l+2}$.

By definition, $a_{2 l+3}$ must maximize the function

$$
U(x)+\left|x-a_{2 l+2}\right|^{\lambda} \quad x \in S^{d} .
$$

Since $U(x)$ has a maximum at $-a_{2 l+2}$ and the function $x \mapsto\left|x-a_{2 l+2}\right|^{\lambda}$ has a unique maximum at $-a_{2 l+2}$, necessarily $a_{2 l+3}=-a_{2 l+2}$. We have proved (2.1) by induction, and (2.2) follows.

\section{The case $0<\lambda<2$}

In this section we present results valid in the range $0<\lambda<2$.

\subsection{First-order asymptotics and uniform distribution}

We describe in this subsection the first-order asymptotic behavior of the sequences $\left(H_{\lambda}\left(\alpha_{N, \lambda}\right)\right)_{N=2}^{\infty}$ and $\left(U_{n}\left(a_{n}\right)\right)_{n=1}^{\infty}$. It is also shown that in the range $0<\lambda<2$, greedy $\lambda$-energy sequences are uniformly distributed on $S^{d}$. 
Theorem 3.1. Assume $0<\lambda<2$, let $d \geq 1$ be arbitrary, and let $\left(a_{n}\right)_{n=0}^{\infty} \subset S^{d}$ be a greedy $\lambda$-energy sequence. The associated sequence of configurations (1.4) satisfies

$$
N(N-1) I_{\lambda}\left(\sigma_{d}\right)<H_{\lambda}\left(\alpha_{N, \lambda}\right)<N^{2} I_{\lambda}\left(\sigma_{d}\right), \quad N \geq 2 .
$$

In particular,

$$
\lim _{N \rightarrow \infty} \frac{H_{\lambda}\left(\alpha_{N, \lambda}\right)}{N^{2}}=I_{\lambda}\left(\sigma_{d}\right) .
$$

For the sequence (1.5) we have

$$
\sigma_{N, \lambda} \stackrel{*}{\longrightarrow} \sigma_{d} .
$$

Proof. We follow the strategy used in the proof of Theorem 1.1 in [16, Ch. V]. Let $U_{n}(x)$ be the function defined in (1.7). In virtue of (1.2),

$$
U_{n}\left(a_{n}\right) \geq U_{n}(x), \quad \text { for all } x \in S^{d}, \quad n \geq 1 .
$$

This implies

$$
H_{\lambda}\left(\alpha_{N, \lambda}\right)=2 \sum_{0 \leq k<i \leq N-1}\left|a_{i}-a_{k}\right|^{\lambda}=2 \sum_{i=1}^{N-1} U_{i}\left(a_{i}\right) \geq 2 \sum_{i=1}^{N-1} U_{i}(x), \quad x \in S^{d} .
$$

In fact, since $U_{1}(x)<U_{1}\left(a_{1}\right)$ for all $x \in S^{d} \backslash\left\{a_{1}\right\}$, we have

$$
\sum_{i=1}^{N-1} U_{i}\left(a_{i}\right)>\sum_{i=1}^{N-1} U_{i}(x), \quad x \in S^{d} \backslash\left\{a_{1}\right\} .
$$

Integrating both sides of (3.5) with respect to $\sigma_{d}$, we get

$$
\begin{aligned}
H_{\lambda}\left(\alpha_{N, \lambda}\right) & >2 \sum_{i=1}^{N-1} \int U_{i}(x) d \sigma_{d}(x) \\
& =2 \sum_{i=1}^{N-1} \sum_{k=0}^{i-1} \int\left|x-a_{k}\right|^{\lambda} d \sigma_{d}(x) \\
& =2 \sum_{i=1}^{N-1} \sum_{k=0}^{i-1} U^{\sigma_{d}}\left(a_{k}\right)
\end{aligned}
$$

where

$$
U^{\sigma_{d}}(y):=\int|x-y|^{\lambda} d \sigma_{d}(x)
$$

is the potential of $\sigma_{d}$. By symmetry,

$$
U^{\sigma_{d}}(y)=I_{\lambda}\left(\sigma_{d}\right) \text { for all } y \in S^{d} .
$$

This and (3.6) imply

$$
H_{\lambda}\left(\alpha_{N, \lambda}\right)>N(N-1) I_{\lambda}\left(\sigma_{d}\right) .
$$

Let $\omega_{N, \lambda}^{*}$ be an $N$-point configuration on $S^{d}$ (with possible point repetitions) that maximizes the energy (1.1) among all such configurations, i.e.,

$$
H_{\lambda}\left(\omega_{N, \lambda}^{*}\right)=\max \left\{H_{\lambda}\left(\omega_{N}\right): \omega_{N} \subset S^{d}, \operatorname{card}\left(\omega_{N}\right)=N \text { counting mult. }\right\} .
$$


Let

$$
\tau_{N}:=\frac{1}{N} \sum_{x \in \omega_{N, \lambda}^{*}} \delta_{x}
$$

Applying Theorem 1.1, we have

$$
H_{\lambda}\left(\alpha_{N, \lambda}\right) \leq H_{\lambda}\left(\omega_{N, \lambda}^{*}\right)=N^{2} I_{\lambda}\left(\tau_{N}\right)<N^{2} I_{\lambda}\left(\sigma_{d}\right) .
$$

This finishes the proof of (3.1) and (3.2).

Let $\sigma$ be a limit point of the sequence $\left(\sigma_{N, \lambda}\right)_{N=1}^{\infty}$ in the weak-star topology. Let $\left(\sigma_{N, \lambda}\right)_{N \in \mathcal{N}}$ be a subsequence that converges to $\sigma$. Then

$$
\lim _{N \in \mathcal{N}} \iint|x-y|^{\lambda} d \sigma_{N, \lambda}(x) d \sigma_{N, \lambda}(y)=\iint|x-y|^{\lambda} d \sigma(x) d \sigma(y) .
$$

This is equivalent to $\lim _{N \in \mathcal{N}} N^{-2} H_{\lambda}\left(\alpha_{N, \lambda}\right)=I_{\lambda}(\sigma)$, hence $I_{\lambda}(\sigma)=I_{\lambda}\left(\sigma_{d}\right)$. By the uniqueness of the maximal distribution, we get $\sigma=\sigma_{d}$. Since any limit point of the sequence $\left(\sigma_{N, \lambda}\right)_{N=1}^{\infty}$ is $\sigma_{d}$, we obtain (3.3).

Remark 3.2. In virtue of (3.1), the sequence $\left(\left(H_{\lambda}\left(\alpha_{N, \lambda}\right)-N^{2} I_{\lambda}\left(\sigma_{d}\right)\right) / N\right)_{N=2}^{\infty}$ is bounded and we have

$$
-I_{\lambda}\left(\sigma_{d}\right)<\frac{H_{\lambda}\left(\alpha_{N, \lambda}\right)-N^{2} I_{\lambda}\left(\sigma_{d}\right)}{N}<0 \quad \text { for all } N \geq 2 .
$$

In the case of the unit circle $d=1$, our later results show that

$$
\lim _{N \rightarrow \infty} \frac{H_{\lambda}\left(\alpha_{N, \lambda}\right)-N^{2} I_{\lambda}\left(\sigma_{1}\right)}{N}=0 .
$$

Our next result concerns the sequence $\left(U_{n}\left(a_{n}\right)\right)_{n=1}^{\infty}$.

Theorem 3.3. Assume $0<\lambda<2$, let $d \geq 1$ be arbitrary, and let $\left(a_{n}\right)_{n=0}^{\infty} \subset S^{d}$ be a greedy $\lambda$-energy sequence. Let $U_{n}$ be the potential (1.7). The following properties hold:

1) For every $n \geq 1$,

$$
U_{n}\left(a_{n}\right) \leq U_{n+1}\left(a_{n+1}\right) \leq U_{n}\left(a_{n}\right)+\left|a_{n+1}-a_{n}\right|^{\lambda} .
$$

2) For every $n \geq 1$, the function $U_{n}$ is not constant s $^{\S}$ on $S^{d}$.

3) For every $n \geq 1$ and $k \geq 0$,

$$
\begin{aligned}
n I_{\lambda}\left(\sigma_{d}\right) & <U_{n}\left(a_{n}\right) \leq n 2^{\lambda} \\
U_{2 k+1}\left(a_{2 k+1}\right) & =U_{2 k}\left(a_{2 k}\right)+2^{\lambda}
\end{aligned}
$$

understanding $U_{0}\left(a_{0}\right)=0$.

4) We have

$$
\lim _{n \rightarrow \infty} \frac{U_{n}\left(a_{n}\right)}{n}=I_{\lambda}\left(\sigma_{d}\right)
$$

§See Remark 3.4 
Proof. Let $n \geq 1$. We have $U_{n+1}(x)=U_{n}(x)+\left|x-a_{n}\right|^{\lambda} \geq U_{n}(x)$ for all $x \in S^{d}$, hence

$$
U_{n}\left(a_{n}\right)=\max _{x \in S^{d}} U_{n}(x) \leq \max _{x \in S^{d}} U_{n+1}(x)=U_{n+1}\left(a_{n+1}\right) .
$$

Taking $x=a_{n+1}$ in (3.4), we get

$$
U_{n}\left(a_{n}\right) \geq U_{n}\left(a_{n+1}\right)=\sum_{k=0}^{n}\left|a_{n+1}-a_{k}\right|^{\lambda}-\left|a_{n+1}-a_{n}\right|^{\lambda}=U_{n+1}\left(a_{n+1}\right)-\left|a_{n+1}-a_{n}\right|^{\lambda} .
$$

So (3.9) is justified.

Suppose that for some $n \geq 1$, the function $U_{n}(x)=\sum_{j=0}^{n-1}\left|x-a_{j}\right|^{\lambda}$ is constant on $S^{d}$. Then, one could construct a periodic greedy $\lambda$-energy sequence with period $n$, repeating indefinitely the cycle $\left(a_{0}, \ldots, a_{n-1}\right)$. But this contradicts (3.3).

Integrating both sides of (3.4) with respect to $\sigma_{d}$, and using the fact that the function $U_{n}\left(a_{n}\right)-U_{n}(x) \geq 0$ is not constant on $S^{d}$, we get

$$
U_{n}\left(a_{n}\right)>\int U_{n}(x) d \sigma_{d}(x)=\sum_{k=0}^{n-1} \int\left|x-a_{k}\right|^{\lambda} d \sigma_{d}(x)=\sum_{k=0}^{n-1} U^{\sigma_{d}}\left(a_{k}\right)=n I_{\lambda}\left(\sigma_{d}\right),
$$

where we applied (3.7) in the last equality. This proves the first inequality in (3.10). Clearly, $U_{1}\left(a_{1}\right)=\left|a_{1}-a_{0}\right|^{\lambda}=2^{\lambda}$. The second inequality in (3.10) then follows from (3.9) and induction.

For each $k \geq 1$, applying (2.2) we get

$$
\begin{aligned}
U_{2 k}\left(a_{2 k}\right) & =U_{2 k}\left(-a_{2 k}\right)=U_{2 k}\left(a_{2 k+1}\right) \\
& =U_{2 k+1}\left(a_{2 k+1}\right)-\left|a_{2 k+1}-a_{2 k}\right|^{\lambda} \\
& =U_{2 k+1}\left(a_{2 k+1}\right)-2^{\lambda} .
\end{aligned}
$$

This justifies (3.11).

Now we prove (3.12). The argument we employ follows the line of reasoning in the proof of Theorem 1.2 in [16, Ch. V]. By (3.10) we have $\frac{U_{n}\left(a_{n}\right)}{n}>I_{\lambda}\left(\sigma_{d}\right)$ for all $n \geq 1$. Let $0<\epsilon<1$, and assume that $m \geq 1$ is a fixed index for which

$$
\frac{U_{m}\left(a_{m}\right)}{m} \geq I_{\lambda}\left(\sigma_{d}\right)+\epsilon
$$

From (3.9) we deduce

$$
U_{i}\left(a_{i}\right) \geq U_{i+1}\left(a_{i+1}\right)-2^{\lambda}, \quad i \geq 1 .
$$

Hence,

$$
\frac{U_{m-1}\left(a_{m-1}\right)}{m} \geq \frac{U_{m}\left(a_{m}\right)-2^{\lambda}}{m} \geq I_{\lambda}\left(\sigma_{d}\right)+\epsilon-\frac{2^{\lambda}}{m} .
$$

Similarly, a repeated application of (3.14) yields

$$
\frac{U_{i}\left(a_{i}\right)}{m} \geq I_{\lambda}\left(\sigma_{d}\right)+\epsilon-(m-i) \frac{2^{\lambda}}{m}, \quad 1 \leq i \leq m .
$$

In particular, we have

$$
\frac{U_{i}\left(a_{i}\right)}{m} \geq I_{\lambda}\left(\sigma_{d}\right)+\frac{\epsilon}{2}, \quad m\left(1-\frac{\epsilon}{2^{\lambda+1}}\right) \leq i \leq m .
$$


Let $\kappa_{m}:=m\left(1-2^{-\lambda-1} \epsilon\right)$.

We have

$$
\begin{aligned}
2^{-1} H_{\lambda}\left(\alpha_{m+1, \lambda}\right) & =\sum_{i=1}^{m} U_{i}\left(a_{i}\right) \\
& =\sum_{1 \leq i<\kappa_{m}} U_{i}\left(a_{i}\right)+\sum_{\kappa_{m} \leq i \leq m} U_{i}\left(a_{i}\right) \\
& \geq \sum_{1 \leq i<\kappa_{m}} i I_{\lambda}\left(\sigma_{d}\right)+\sum_{\kappa_{m} \leq i \leq m} m\left(I_{\lambda}\left(\sigma_{d}\right)+\frac{\epsilon}{2}\right)
\end{aligned}
$$

where we applied the first inequality in (3.10) and (3.15). This easily implies

$$
\frac{H_{\lambda}\left(\alpha_{m+1, \lambda}\right)}{(m+1)^{2}} \geq I_{\lambda}\left(\sigma_{d}\right) \frac{\left(\left\lfloor\kappa_{m}\right\rfloor-1\right)\left\lfloor\kappa_{m}\right\rfloor}{(m+1)^{2}}+\left(I_{\lambda}\left(\sigma_{d}\right)+\frac{\epsilon}{2}\right) \frac{2 m\left(m-\left\lfloor\kappa_{m}\right\rfloor\right)}{(m+1)^{2}}
$$

where $\lfloor\cdot\rfloor$ is the floor function. Thus, we have shown that (3.13) implies (3.16).

Assume that there are infinitely many indices $m$ that satisfy (3.13), and they form the subsequence $\mathcal{N}$. Then, along this subsequence, the right-hand side of (3.16) approaches the value

$$
I_{\lambda}\left(\sigma_{d}\right)\left(1+\frac{\epsilon^{2}}{2^{2(\lambda+1)}}\right)+\frac{\epsilon^{2}}{2^{\lambda+1}} .
$$

However, according to (3.2) the left-hand side of (3.16) has limit $I_{\lambda}\left(\sigma_{d}\right)$ as $m \rightarrow \infty$ along $\mathcal{N}$. This contradiction shows that (3.13) is only valid for finitely many $m$ 's, and concludes the proof of (3.12).

Formula (3.12) can also be obtained as an application of (3.3) and Theorem 1.2 in [18. It also follows from Theorem 2.1 in 12 .

Remark 3.4. Property 2) in Theorem 3.3 is not valid for $\lambda=2$. Indeed, we will see that in this case $U_{2 k} \equiv 4 k$ on $S^{d}$ for every $k \geq 1$.

\subsection{Binary representation of the energy on the unit circle}

The rest of Section 3 is devoted to the analysis of greedy $\lambda$-energy sequences on the unit circle $S^{1}$.

Lemma 3.5. Assume $0<\lambda \leq 1$, and let $z_{1}$ and $z_{2}$ be two distinct points on $S^{1}$. Consider the function

$$
\left|z-z_{1}\right|^{\lambda}+\left|z-z_{2}\right|^{\lambda}, \quad z \in S^{1} .
$$

Let $\gamma$ be any of the two closed arcs on $S^{1}$ that connect $z_{1}$ and $z_{2}$. On $\gamma$, the function in (3.17) has a unique maximum, which is attained at the middle point of the arc $\gamma$.

Proof. Without loss of generality, we assume that $z_{1}=\overline{z_{2}}$. Hence, $z_{1}=e^{i \phi}$ and $z_{2}=e^{-i \phi}$, where $0<\phi<\pi$. Define the function

$$
f(\theta):=\left|e^{i \theta}-e^{i \phi}\right|^{\lambda}+\left|e^{i \theta}-e^{-i \phi}\right|^{\lambda}, \quad-\phi \leq \theta \leq \phi .
$$

We have

$$
f(\theta)=2^{\lambda} g(\theta)
$$


where

$$
g(\theta):=\sin ^{\lambda}\left(\frac{\phi-\theta}{2}\right)+\sin ^{\lambda}\left(\frac{\phi+\theta}{2}\right), \quad-\phi \leq \theta \leq \phi .
$$

The derivative of $g$ is

$$
g^{\prime}(\theta)=\frac{\lambda}{2}\left(\sin ^{\lambda-1}\left(\frac{\phi+\theta}{2}\right) \cos \left(\frac{\phi+\theta}{2}\right)-\sin ^{\lambda-1}\left(\frac{\phi-\theta}{2}\right) \cos \left(\frac{\phi-\theta}{2}\right)\right),
$$

valid for $\theta \in(-\phi, \phi)$. Then,

$g^{\prime \prime}(\theta)=-\frac{\lambda}{4}\left[\sin ^{\lambda-2}\left(\frac{\phi+\theta}{2}\right)\left(1-\lambda \cos ^{2}\left(\frac{\phi+\theta}{2}\right)\right)+\sin ^{\lambda-2}\left(\frac{\phi-\theta}{2}\right)\left(1-\lambda \cos ^{2}\left(\frac{\phi-\theta}{2}\right)\right)\right]$,

also for $\theta \in(-\phi, \phi)$.

Assume that $0<\lambda \leq 1$. Since $0<\frac{\phi \pm \theta}{2}<\pi$, we have $1-\lambda \cos ^{2}\left(\frac{\phi \pm \theta}{2}\right)>0$. We also have $\sin ^{\lambda-2}((\phi \pm \theta) / 2)>0$, therefore

$$
g^{\prime \prime}(\theta)<0 \quad \text { for all } \theta \in(-\phi, \phi) .
$$

It follows that $g$ and $f$ attain their maximum value on the interval $-\phi \leq \theta \leq \phi$ only at $\theta=0$.

It is interesting to remark that the property stated in Lemma 3.5 is not valid in the range $1<\lambda<2$. However, we have the following result due to Stolarsky, see [19, Theorem 1.2].

Lemma 3.6 (Stolarsky [19]). Let $e_{1}, \ldots, e_{n}$ be $n$ equally spaced points on $S^{1}$, and let $0<\lambda<2$. The function $\sum_{i=1}^{n}\left|z-e_{i}\right|^{\lambda}, z \in S^{1}$, attains its maximum value only at the midpoints of the arcs between consecutive $e_{i}$.

With Lemma 3.6 at hand, we can give a geometric description of greedy $\lambda$-energy sequences on $S^{1}$ for $0<\lambda<2$. We remind the reader that throughout this work, we always assume that greedy $\lambda$-energy sequences on $S^{1}$ have initial point $a_{0}=1$.

We can use the same argument employed in the proof of [1, Theorem 5] to prove our next result. We reproduce the argument for convenience of the reader. In the rest of this work, we adopt the following notation. If $\alpha=\left(a_{0}, \ldots, a_{k}\right)$ and $\beta=\left(b_{0}, \ldots, b_{l}\right)$, then $(\alpha, \beta)$ will indicate the finite sequence $\left(a_{0}, \ldots, a_{k}, b_{0}, \ldots, b_{l}\right)$.

Lemma 3.7. Let $0<\lambda<2$, and let $\left(a_{n}\right)_{n=0}^{\infty}$ be a greedy $\lambda$-energy sequence on $S^{1}$. For each $N \geq 1$, let $\alpha_{N}=\left(a_{0}, \ldots, a_{N-1}\right)$. Then, for all $n \geq 0$, the configuration $\alpha_{2^{n}}$ consists of the $2^{n}$-th roots of unity, and we have

$$
\alpha_{2^{n+1}}=\left(\alpha_{2^{n}}, \rho \beta_{2^{n}}\right),
$$

where $\rho$ is a solution of $z^{2^{n}}=-1$, and $\beta_{2^{n}}$ is the $2^{n}$-th section of a greedy $\lambda$-energy sequence on $S^{1}$ with initial point $b_{0}=1$.

Proof. We proceed by induction. For $n=0$, both statements are certainly true since $\alpha_{2}=$ $(1,-1)$. Now assume that the $2^{n}$-th section of any greedy $\lambda$-energy sequence on $S^{1}$ consists of the $2^{n}$-th roots of unity, and let us prove (3.18). This, in turn, implies that the section $\alpha_{2^{n+1}}$ consists of the $2^{n+1}$-st roots of unity, which completes the induction argument.

Set $m=2^{n}$. We want to prove that

$$
a_{m+k}=\rho b_{k}, \quad 0 \leq k \leq 2^{n}-1,
$$


where $\rho$ is a solution of $z^{2^{n}}=-1$ and $\left(b_{0}, \ldots, b_{2^{n}-1}\right)$ is the $2^{n}$-th section of a greedy sequence. By the induction hypothesis, $\alpha_{m}=\left(a_{0}, \ldots, a_{m-1}\right)$ consists of the $2^{n}$-th roots of unity. By Lemma 3.6, the function

$$
\sum_{\ell=0}^{m-1}\left|a_{\ell}-z\right|^{\lambda}
$$

is maximized precisely when $z=a_{m}$ is the midpoint of one of the arcs determined by two points in $\alpha_{m}$ lying consecutively on $S^{1}$. Therefore, the point $\rho:=a_{m}$ is a $2^{n}$-th root of -1 . If we set $b_{0}=1$, then we have proved (3.19) for $k=0$.

As a side remark, observe that if $0<\lambda \leq 1$, then we can apply Lemma 3.5 to justify that $\rho=a_{m}$ is a $2^{n}$-th root of -1 . Indeed, if we assume, to fix notation, that $0 \leq \arg \left(a_{m}\right) \leq 2 \pi / m$, we express the potential as follows

$$
\begin{gathered}
\sum_{\ell=0}^{m-1}\left|a_{\ell}-z\right|^{\lambda}=\sum_{\ell=0}^{m-1}\left|e^{2 \pi i \ell / m}-z\right|^{\lambda} \\
=\sum_{\ell=0}^{(m-2) / 2}\left(\left|e^{-2 \pi i \ell / m}-z\right|^{\lambda}+\left|e^{2 \pi i(1+\ell) / m}-z\right|^{\lambda}\right) .
\end{gathered}
$$

By Lemma 3.5. each expression $\left|e^{-2 \pi i \ell / m}-z\right|^{\lambda}+\left|e^{2 \pi i(1+\ell) / m}-z\right|^{\lambda}$ has a unique maximum on the $\operatorname{arc} \arg (z) \in[0,2 \pi / m]$ at $\arg (z)=\pi / m$. Hence $\arg \left(a_{m}\right)=\pi / m$ and $a_{m}=\rho$ is a $2^{n}$-th root of -1 .

We prove (3.19) by induction on $k$. Let $0 \leq k<2^{n}-1$ be fixed. We assume that $a_{m+j}=\rho b_{j}$, $0 \leq j \leq k$, where $\left(b_{0}, \ldots, b_{k}\right)$ is the $(k+1)$-st section of a greedy $\lambda$-energy sequence on $S^{1}$, and we prove that if $b_{k+1}$ is defined by $a_{m+k+1}=\rho b_{k+1}$, then

$$
\sum_{j=0}^{k}\left|b_{k+1}-b_{j}\right|^{\lambda}=\max _{|z|=1} \sum_{j=0}^{k}\left|z-b_{j}\right|^{\lambda} .
$$

Let $U_{n}(z)$ be the discrete potential (1.7). By definition, $a_{m+k+1}$ satisfies

$$
U_{m+k+1}\left(a_{m+k+1}\right)=\max _{|z|=1} U_{m+k+1}(z)
$$

and we have

$$
\begin{aligned}
U_{m+k+1}\left(a_{m+k+1}\right) & =U_{m+k+1}\left(\rho b_{k+1}\right)=\max _{|z|=1} U_{m+k+1}(z) \\
& =\max _{|z|=1} U_{m+k+1}(\rho z) \\
& =\max _{|z|=1}\left(U_{m}(\rho z)+\sum_{j=0}^{k}\left|\rho z-\rho b_{j}\right|^{\lambda}\right) \\
& =\max _{|z|=1}\left(U_{m}(\rho z)+\sum_{j=0}^{k}\left|z-b_{j}\right|^{\lambda}\right) \\
& =\max _{|z|=1} U_{m}(\rho z)+\max _{|z|=1} \sum_{j=0}^{k}\left|z-b_{j}\right|^{\lambda}
\end{aligned}
$$


where the last equality follows from the fact that both expressions $U_{m}(\rho z)$ and $\sum_{j=0}^{k}\left|z-b_{j}\right|^{\lambda}$ are maximized at a $2^{n}$-th root of unity. Therefore,

$$
\begin{aligned}
U_{m+k+1}\left(\rho b_{k+1}\right) & =U_{m}\left(\rho b_{k+1}\right)+\sum_{j=0}^{k}\left|b_{k+1}-b_{j}\right|^{\lambda} \\
& =\max _{|z|=1} U_{m}(\rho z)+\max _{|z|=1} \sum_{j=0}^{k}\left|z-b_{j}\right|^{k}
\end{aligned}
$$

and (3.20) follows. This concludes the proof.

We deduce from Lemma 3.7 the following consequence. Assume $0<\lambda<2$ and $\left(a_{n}\right)_{n=0}^{\infty}$ is a greedy $\lambda$-energy sequence on $S^{1}$. If $2^{n}$ is the largest power of 2 that does not exceed $N$, then

$$
\alpha_{N}=\left(\alpha_{2^{n}}, \rho \beta_{N-2^{n}}\right),
$$

where $\rho$ satisfies $z^{2^{n}}=-1$ and $\beta_{N-2^{n}}$ is the section of order $N-2^{n}$ of a greedy $\lambda$-energy sequence on $S^{1}$.

Define

$$
\mathcal{L}_{\lambda}(N):=N 2^{\lambda} \sum_{k=1}^{N-1}\left(\sin \frac{\pi k}{N}\right)^{\lambda}, \quad N \geq 1 .
$$

In the case $N=1$, we understand $\mathcal{L}_{\lambda}(1)=0$. The reader can check that this expression is the $\lambda$-energy of $N$ equally spaced points on $S^{1}$. In the range $0<\lambda<2$, this value is the largest $\lambda$-energy value for an $N$-point configuration on $S^{1}$, i.e.,

$$
\mathcal{L}_{\lambda}(N)=\sup \left\{H_{\lambda}\left(\omega_{N}\right): \omega_{N} \subset S^{1}, \operatorname{card}\left(\omega_{N}\right)=N\right\}, \quad N \geq 2,
$$

see Theorem 2.3.3 and Remark 2.3.4 in [3].

In our next result, we describe the $\lambda$-energy of the $N$-th section of a greedy $\lambda$-energy sequence, in terms of the binary representation of $N$.

Lemma 3.8. Let $0<\lambda<2$, and let $\left(a_{n}\right)_{n=0}^{\infty}$ be a greedy $\lambda$-energy sequence on $S^{1}$. Assume that $N \geq 2$ has the binary representation

$$
N=2^{n_{1}}+\cdots+2^{n_{p}}, \quad n_{1}>n_{2}>\cdots>n_{p} \geq 0 .
$$

Then, the $\lambda$-energy of $\alpha_{N}=\left(a_{n}\right)_{n=0}^{N-1}$ is given by

$$
H_{\lambda}\left(\alpha_{N}\right)=\sum_{k=1}^{p-1}\left(\sum_{j=k+1}^{p} 2^{n_{j}-n_{k}}\right) \mathcal{L}_{\lambda}\left(2^{n_{k}+1}\right)+\sum_{k=1}^{p}\left(1-\sum_{j=k+1}^{p} 2^{n_{j}-n_{k}+1}\right) \mathcal{L}_{\lambda}\left(2^{n_{k}}\right),
$$

understanding $\sum_{i_{1}}^{i_{2}}$ as empty sum if $i_{2}<i_{1}$.

Proof. We use induction on $p$, the number of terms in the binary expansion of $N$. If $p=1$, then $N=2^{n}$, so by Lemma 3.7 we have $H_{\lambda}\left(\alpha_{2^{n}}\right)=\mathcal{L}_{\lambda}\left(2^{n}\right)$, hence (3.25) holds in this case.

For a fixed $p \geq 1$, assume that (3.25) is valid for every integer $N \geq 2$ with $p$ terms in its binary expansion, and let $\widetilde{N}=2^{n_{1}}+\cdots+2^{n_{p}}+2^{n_{p+1}}, n_{1}>\cdots>n_{p}>n_{p+1} \geq 0$. Then, $2^{n_{1}}$ is the largest power of 2 not greater than $N$, so by (3.21), we have

$$
\alpha_{\widetilde{N}}=\left(\alpha_{2^{n_{1}}}, \rho \beta_{\widetilde{N}-2^{n_{1}}}\right)
$$


where $\rho$ satisfies $z^{2^{n_{1}}}=-1$, and $\beta_{\widetilde{N}-2^{n_{1}}}$ is the section of order $\widetilde{N}-2^{n_{1}}=2^{n_{2}}+\cdots+2^{n_{p+1}}$ of a greedy $\lambda$-energy sequence. Let us write $A=\alpha_{2^{n_{1}}}, B=\beta_{\widetilde{N}-2^{n_{1}}}$.

Assume first that $\operatorname{card}(B)=\widetilde{N}-2^{n_{1}} \geq 2$. Since $A$ and $B$ are disjoint, we have

$$
H_{\lambda}\left(\alpha_{\widetilde{N}}\right)=H_{\lambda}(A)+H_{\lambda}(B)+2 \sum_{y \in B} \sum_{x \in A}|x-y|^{\lambda} .
$$

The configuration $A$ is formed by equally spaced points, so

$$
H_{\lambda}(A)=\mathcal{L}_{\lambda}\left(2^{n_{1}}\right) .
$$

We also know by Lemma 3.7 that every point $y \in B$ is a midpoint of an arc whose endpoints are neighboring points in $A$. Therefore, for each $y \in B$, we have

$$
\sum_{x \in A}|x-y|^{\lambda}=2^{-n_{1}-1} \mathcal{L}_{\lambda}\left(2^{n_{1}+1}\right)-2^{-n_{1}} \mathcal{L}_{\lambda}\left(2^{n_{1}}\right),
$$

consequently,

$$
\begin{aligned}
2 \sum_{y \in B} \sum_{x \in A}|x-y|^{\lambda} & =2 \operatorname{card}(B)\left(2^{-n_{1}-1} \mathcal{L}_{\lambda}\left(2^{n_{1}+1}\right)-2^{-n_{1}} \mathcal{L}_{\lambda}\left(2^{n_{1}}\right)\right) \\
& =\sum_{j=2}^{p+1} 2^{n_{j}-n_{1}} \mathcal{L}_{\lambda}\left(2^{n_{1}+1}\right)-\sum_{j=2}^{p+1} 2^{n_{j}-n_{1}+1} \mathcal{L}_{\lambda}\left(2^{n_{1}}\right) .
\end{aligned}
$$

We obtain

$$
H_{\lambda}\left(\alpha_{\widetilde{N}}\right)=\left(\sum_{j=2}^{p+1} 2^{n_{j}-n_{1}}\right) \mathcal{L}_{\lambda}\left(2^{n_{1}+1}\right)+\left(1-\sum_{j=2}^{p+1} 2^{n_{j}-n_{1}+1}\right) \mathcal{L}_{\lambda}\left(2^{n_{1}}\right)+H_{\lambda}(B) .
$$

By the induction hypothesis, the energy of $B$ is given by

$$
H_{\lambda}(B)=\sum_{k=2}^{p}\left(\sum_{j=k+1}^{p+1} 2^{n_{j}-n_{k}}\right) \mathcal{L}_{\lambda}\left(2^{n_{k}+1}\right)+\sum_{k=2}^{p+1}\left(1-\sum_{j=k+1}^{p+1} 2^{n_{j}-n_{k}+1}\right) \mathcal{L}_{\lambda}\left(2^{n_{k}}\right) .
$$

From the previous two expressions we deduce

$$
H_{\lambda}\left(\alpha_{\widetilde{N}}\right)=\sum_{k=1}^{p}\left(\sum_{j=k+1}^{p+1} 2^{n_{j}-n_{k}}\right) \mathcal{L}_{\lambda}\left(2^{n_{k}+1}\right)+\sum_{k=1}^{p+1}\left(1-\sum_{j=k+1}^{p+1} 2^{n_{j}-n_{k}+1}\right) \mathcal{L}_{\lambda}\left(2^{n_{k}}\right),
$$

which proves (3.25) for $\widetilde{N}$, in the $\operatorname{case} \operatorname{card}(B)=\widetilde{N}-2^{n_{1}} \geq 2$. If $\operatorname{card}(B)=1$, then $\widetilde{N}=2^{n_{1}}+1$, $n_{1} \geq 1$, so

$$
\begin{aligned}
H_{\lambda}\left(\alpha_{\widetilde{N}}\right) & =H_{\lambda}(A)+2 \sum_{x \in A}|x-\rho|^{\lambda} \\
& =2^{-n_{1}} \mathcal{L}_{\lambda}\left(2^{n_{1}+1}\right)+\left(1-2^{-n_{1}+1}\right) \mathcal{L}_{\lambda}\left(2^{n_{1}}\right)
\end{aligned}
$$

which coincides with (3.25). 
We define now the quantities

$$
\mathcal{U}_{\lambda}(N):=\sum_{k=0}^{N-1}\left|e^{i \pi / N}-e^{2 \pi i k / N}\right|^{\lambda}, \quad N \geq 1
$$

This represents the discrete potential of $N$ equally spaced points evaluated at the midpoint of one of the arcs between two adjacent points. If $e_{1}, \ldots, e_{N}$ are $N$ equally spaced points on the unit circle, then

$$
\sum_{i=1}^{N-1}\left|e_{i}-e_{N}\right|^{\lambda}=\frac{\mathcal{L}_{\lambda}(N)}{N}
$$

From this relation it is easy to deduce the identity

$$
\mathcal{U}_{\lambda}(N)=\frac{\mathcal{L}_{\lambda}(2 N)}{2 N}-\frac{\mathcal{L}_{\lambda}(N)}{N} .
$$

Lemma 3.9. Let $0<\lambda<2$, and let $\left(a_{n}\right)_{n=0}^{\infty}$ be a greedy $\lambda$-energy sequence on $S^{1}$. If $N \geq 1$ has the binary representation (3.24), then the function $U_{N}(x)$ (1.7) satisfies

$$
U_{N}\left(a_{N}\right)=\sum_{k=1}^{p} \mathcal{U}_{\lambda}\left(2^{n_{k}}\right) .
$$

Proof. The proof is also by induction on $p$. If $p=1$ and $N=2^{n}$, then by Lemma 3.7 the points in $\alpha_{N}=\left(a_{0}, \ldots, a_{N-1}\right)$ are the $N$-th roots of unity. The point $a_{N}$ is the midpoint of one of the arcs between adjacent points in $\alpha_{N}$, hence $U_{N}\left(a_{N}\right)=\mathcal{U}_{\lambda}(N)$.

For a fixed $p \geq 1$, assume as induction hypothesis that (3.28) is valid for every greedy sequence and every $N \geq 1$ with binary representation of length $p$. Let $\widetilde{N}=2^{n_{1}}+\cdots+2^{n_{p}}+2^{n_{p+1}}$, $n_{1}>\cdots>n_{p}>n_{p+1} \geq 0$. Then, as in the proof of Lemma 3.8 we can write

$$
\alpha_{\widetilde{N}}=\left(\alpha_{2^{n_{1}}}, \rho \beta_{\widetilde{N}-2^{n_{1}}}\right),
$$

where $\rho$ satisfies $z^{2^{n_{1}}}=-1$, and $\beta_{\widetilde{N}-2^{n_{1}}}$ is the section of order $\widetilde{N}-2^{n_{1}}$ of a greedy $\lambda$-energy sequence $\left(b_{k}\right)_{k=0}^{\infty}$. So $a_{k}=\rho b_{k-2^{n_{1}}}, 2^{n_{1}} \leq k \leq \widetilde{N}$, where $\left(b_{0}, \ldots, b_{\widetilde{N}-2^{n_{1}}}\right)=\beta_{\widetilde{N}-2^{n_{1}}+1}$. We have

$$
U_{\widetilde{N}}\left(a_{\widetilde{N}}\right)=U_{2^{n_{1}}}\left(a_{\widetilde{N}}\right)+\sum_{k=2^{n_{1}}}^{\widetilde{N}-1}\left|a_{k}-a_{\widetilde{N}}\right|^{\lambda} .
$$

The point $a_{\widetilde{N}}$ is the midpoint of one of the arcs between two adjacent points in $\alpha_{2^{n_{1}}}$, hence

$$
U_{2^{n_{1}}}\left(a_{\widetilde{N}}\right)=\mathcal{U}_{\lambda}\left(2^{n_{1}}\right)
$$

We also have

$$
\sum_{k=2^{n_{1}}}^{\widetilde{N}-1}\left|a_{k}-a_{\widetilde{N}}\right|^{\lambda}=\sum_{k=2^{n_{1}}}^{\widetilde{N}-1}\left|\rho b_{k-2^{n_{1}}}-\rho b_{\widetilde{N}-2^{n_{1}}}\right|^{\lambda}=\sum_{k=0}^{\widetilde{N}-2^{n_{1}}-1}\left|b_{k}-b_{\widetilde{N}-2^{n_{1}}}\right|^{\lambda}=U_{M}\left(b_{M}\right),
$$

where $M:=\widetilde{N}-2^{n_{1}}$. The number $M=2^{n_{2}}+\cdots+2^{n_{p+1}}$ has a binary representation of length $p$, so by induction hypothesis we obtain

$$
U_{M}\left(b_{M}\right)=\sum_{k=2}^{p+1} \mathcal{U}_{\lambda}\left(2^{n_{k}}\right)
$$

In conclusion, $U_{\widetilde{N}}\left(a_{\widetilde{N}}\right)=\sum_{k=1}^{p+1} \mathcal{U}_{\lambda}\left(2^{n_{k}}\right)$, which finishes the proof of (3.28). 
We can write a more convenient expression for $\mathcal{U}_{\lambda}\left(2^{n}\right)$. From (3.27) and (3.22) we get

$$
\mathcal{U}_{\lambda}\left(2^{n}\right)=\frac{\mathcal{L}_{\lambda}\left(2^{n+1}\right)}{2^{n+1}}-\frac{\mathcal{L}_{\lambda}\left(2^{n}\right)}{2^{n}}=2^{\lambda} \sum_{k=0}^{2^{n}-1} \sin ^{\lambda}\left(\frac{(2 k+1) \pi}{2^{n+1}}\right) .
$$

\subsection{Second-order asymptotics on the unit circle}

Let $\zeta(s):=\sum_{n=1}^{\infty} \frac{1}{n^{s}}, \operatorname{Re}(s)>1$, be the classical Riemann zeta function. We also use $\zeta(s)$ to denote the meromorphic continuation of this function to the entire complex plane. Recall that this function has a simple pole at $s=1$, it is analytic on $\mathbb{C} \backslash\{1\}$, takes negative values on the interval $(-2,1)$, and it has trivial zeros at the points $s=-2 n, n \geq 1$.

Recall the expression

$$
I_{\lambda}\left(\sigma_{1}\right)=\frac{2^{\lambda} \Gamma((1+\lambda) / 2)}{\sqrt{\pi} \Gamma(1+\lambda / 2)} .
$$

Lemma 3.10. Let $0<\lambda<2$. For the sequence (3.26) we have

$$
\begin{gathered}
\lim _{N \rightarrow \infty} \frac{\mathcal{U}_{\lambda}(N)}{N}=I_{\lambda}\left(\sigma_{1}\right), \\
\lim _{N \rightarrow \infty} \frac{\mathcal{U}_{\lambda}(N)-N I_{\lambda}\left(\sigma_{1}\right)}{N^{-\lambda}}=\left(2^{-\lambda}-1\right)(2 \pi)^{\lambda} 2 \zeta(-\lambda) .
\end{gathered}
$$

Proof. The following asymptotic formula, valid for $0<\lambda<2$, follows from [4, Theorem 1.1] (take $s=-\lambda$ and $p=0$ in that result):

$$
\mathcal{L}_{\lambda}(N)=I_{\lambda}\left(\sigma_{1}\right) N^{2}+(2 \pi)^{\lambda} 2 \zeta(-\lambda) N^{1-\lambda}+\mathcal{O}_{\lambda}\left(N^{-1-\lambda}\right), \quad N \rightarrow \infty .
$$

Applying (3.27) and the first two terms in (3.31), we obtain (3.29):

$$
\lim _{N \rightarrow \infty} \frac{\mathcal{U}_{\lambda}(N)}{N}=\lim _{N \rightarrow \infty}\left(2 \frac{\mathcal{L}_{\lambda}(2 N)}{(2 N)^{2}}-\frac{\mathcal{L}_{\lambda}(N)}{N^{2}}\right)=2 I_{\lambda}\left(\sigma_{1}\right)-I_{\lambda}\left(\sigma_{1}\right)=I_{\lambda}\left(\sigma_{1}\right) .
$$

Similarly, we can write

$$
\frac{\mathcal{U}_{\lambda}(N)-N I_{\lambda}\left(\sigma_{1}\right)}{N^{-\lambda}}=2^{-\lambda} \frac{\mathcal{L}_{\lambda}(2 N)-(2 N)^{2} I_{\lambda}\left(\sigma_{1}\right)}{(2 N)^{1-\lambda}}-\frac{\mathcal{L}_{\lambda}(N)-N^{2} I_{\lambda}\left(\sigma_{1}\right)}{N^{1-\lambda}} .
$$

Letting $N \rightarrow \infty$ and using (3.31), we get (3.30).

Theorem 3.11. Let $0<\lambda<2$, and let $\left(a_{n}\right)_{n=0}^{\infty} \subset S^{1}$ be a greedy $\lambda$-energy sequence. Then, the sequence $\left(U_{N}\left(a_{N}\right)-N I_{\lambda}\left(\sigma_{1}\right)\right)_{N=1}^{\infty}$ is bounded and divergent. For each $N \geq 1$,

$$
0<U_{N}\left(a_{N}\right)-N I_{\lambda}\left(\sigma_{1}\right)<I_{\lambda}\left(\sigma_{1}\right) .
$$

These inequalities are sharp since we have

$$
\begin{aligned}
& \liminf _{N \rightarrow \infty}\left(U_{N}\left(a_{N}\right)-N I_{\lambda}\left(\sigma_{1}\right)\right)=0, \\
& \limsup _{N \rightarrow \infty}\left(U_{N}\left(a_{N}\right)-N I_{\lambda}\left(\sigma_{1}\right)\right)=I_{\lambda}\left(\sigma_{1}\right) .
\end{aligned}
$$


Proof. We know from (3.10) that $U_{N}\left(a_{N}\right)-N I_{\lambda}\left(\sigma_{1}\right)>0$ for all $N \geq 1$. This is the first inequality in (3.32).

If we take $N=2^{n}, n \rightarrow \infty$, and apply (3.30), we get

$$
\lim _{n \rightarrow \infty}\left(U_{2^{n}}\left(a_{2^{n}}\right)-2^{n} I_{\lambda}\left(\sigma_{1}\right)\right)=\lim _{n \rightarrow \infty}\left(\mathcal{U}_{\lambda}\left(2^{n}\right)-2^{n} I_{\lambda}\left(\sigma_{1}\right)\right)=0 .
$$

This and the first inequality in (3.32) imply (3.33).

Consider the subsequence

$$
\kappa_{n}:=\sum_{k=0}^{n-1} 2^{k}=2^{n}-1, \quad n \geq 1 .
$$

We claim that the sequence $\left(U_{\kappa_{n}}\left(a_{\kappa_{n}}\right)-\kappa_{n} I_{\lambda}\left(\sigma_{1}\right)\right)_{n=1}^{\infty}$ is strictly increasing. Indeed, according to Lemma 3.9, we have

$$
\begin{aligned}
U_{\kappa_{n+1}}\left(a_{\kappa_{n+1}}\right)-\kappa_{n+1} I_{\lambda}\left(\sigma_{1}\right) & =\sum_{k=0}^{n}\left(\mathcal{U}_{\lambda}\left(2^{k}\right)-2^{k} I_{\lambda}\left(\sigma_{1}\right)\right) \\
& =U_{\kappa_{n}}\left(a_{\kappa_{n}}\right)-\kappa_{n} I_{\lambda}\left(\sigma_{1}\right)+\mathcal{U}_{\lambda}\left(2^{n}\right)-2^{n} I_{\lambda}\left(\sigma_{1}\right)
\end{aligned}
$$

and $\mathcal{U}_{\lambda}\left(2^{n}\right)-2^{n} I_{\lambda}\left(\sigma_{1}\right)=U_{2^{n}}\left(a_{2^{n}}\right)-2^{n} I_{\lambda}\left(\sigma_{1}\right)>0$ for each $n \geq 1$.

Using (3.27), we obtain

$$
U_{\kappa_{n}}\left(a_{\kappa_{n}}\right)=\sum_{k=0}^{n-1} \mathcal{U}_{\lambda}\left(2^{k}\right)=\sum_{k=0}^{n-1}\left(\frac{\mathcal{L}_{\lambda}\left(2^{k+1}\right)}{2^{k+1}}-\frac{\mathcal{L}_{\lambda}\left(2^{k}\right)}{2^{k}}\right)=\frac{\mathcal{L}_{\lambda}\left(2^{n}\right)}{2^{n}},
$$

(recall that $\left.\mathcal{L}_{\lambda}(1)=0\right)$ so

$$
\begin{aligned}
U_{\kappa_{n}}\left(a_{\kappa_{n}}\right)-\kappa_{n} I_{\lambda}\left(\sigma_{1}\right) & =\frac{\mathcal{L}_{\lambda}\left(2^{n}\right)}{2^{n}}-2^{n} I_{\lambda}\left(\sigma_{1}\right)+I_{\lambda}\left(\sigma_{1}\right) \\
& =\frac{\mathcal{L}_{\lambda}\left(2^{n}\right)-2^{2 n} I_{\lambda}\left(\sigma_{1}\right)}{2^{n}}+I_{\lambda}\left(\sigma_{1}\right) .
\end{aligned}
$$

In virtue of (3.31), we have $\lim _{N \rightarrow \infty}\left(\mathcal{L}_{\lambda}(N)-N^{2} I_{\lambda}\left(\sigma_{1}\right)\right) / N=0$, hence the previous identity gives

$$
\lim _{n \rightarrow \infty}\left(U_{\kappa_{n}}\left(a_{\kappa_{n}}\right)-\kappa_{n} I_{\lambda}\left(\sigma_{1}\right)\right)=I_{\lambda}\left(\sigma_{1}\right) .
$$

By monotonicity, this implies

$$
U_{\kappa_{n}}\left(a_{\kappa_{n}}\right)-\kappa_{n} I_{\lambda}\left(\sigma_{1}\right)<I_{\lambda}\left(\sigma_{1}\right), \quad \text { for all } n \geq 1
$$

If $N$ has the binary expansion (3.24), applying (3.28) we obtain

$$
\begin{aligned}
U_{N}\left(a_{N}\right)-N I_{\lambda}\left(\sigma_{1}\right) & =\sum_{k=1}^{p}\left(\mathcal{U}_{\lambda}\left(2^{n_{k}}\right)-2^{n_{k}} I_{\lambda}\left(\sigma_{1}\right)\right) \\
& \leq \sum_{j=0}^{n_{1}}\left(\mathcal{U}_{\lambda}\left(2^{j}\right)-2^{j} I_{\lambda}\left(\sigma_{1}\right)\right) \\
& =U_{\kappa_{n}}\left(a_{\kappa_{n}}\right)-\kappa_{n} I_{\lambda}\left(\sigma_{1}\right) \\
& <I_{\lambda}\left(\sigma_{1}\right),
\end{aligned}
$$

where $n=n_{1}+1$. This justifies the second inequality in (3.32).

Finally, (3.34) follows from (3.32) and (3.35). 


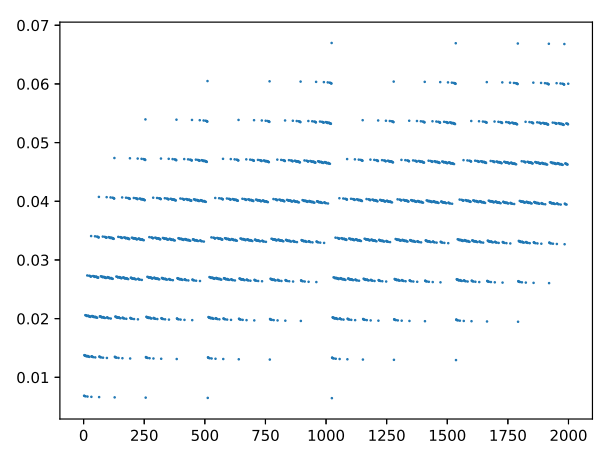

(a) $\lambda=0.01$

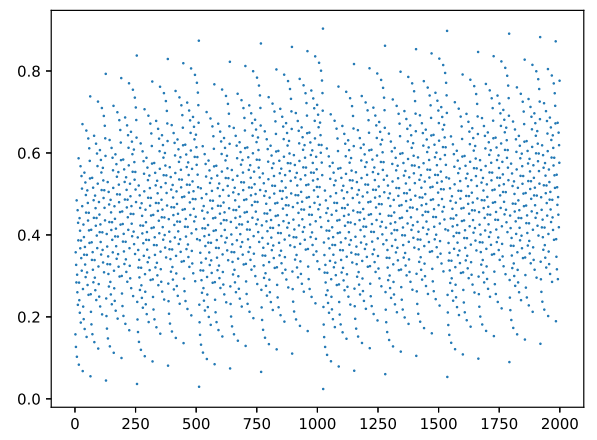

(c) $\lambda=0.3$

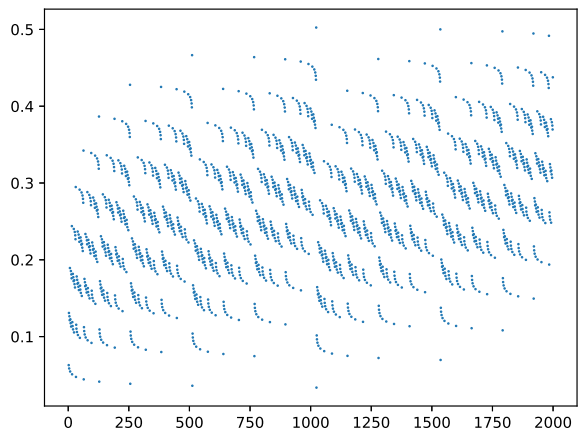

(b) $\lambda=0.1$

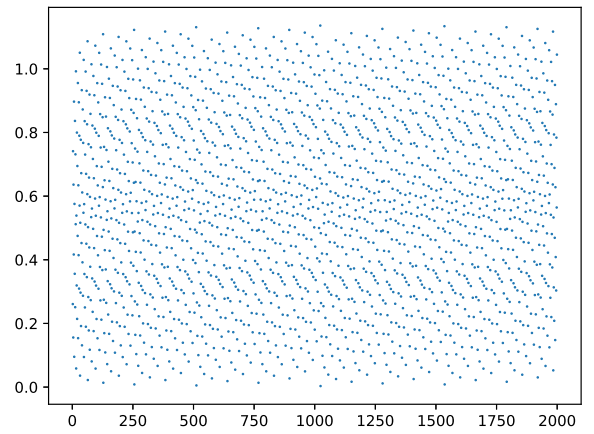

(d) $\lambda=0.7$

Figure 1: Plots of sequences $\left(U_{N}\left(a_{N}\right)-N I_{\lambda}\left(\sigma_{1}\right)\right)$ for $0<\lambda<1$ and $1 \leq N \leq 2000$.

Remark 3.12. Interestingly, the two very close sequences $\left(2^{n}\right)$ and $\left(2^{n}-1\right)$ provide the liminf and limsup of the sequence $\left(U_{N}\left(a_{N}\right)-N I_{\lambda}\left(\sigma_{1}\right)\right)$, respectively.

In Figures 1 and 2 we present plots of the sequences analyzed in Theorem 3.11, for different values of $\lambda \in(0,2)$.

Our goal now is the study of the second-order asymptotics of the energy $H_{\lambda}\left(\alpha_{N, \lambda}\right)$ in the range $0<\lambda<2$. We need to subdivide the analysis into three cases: $0<\lambda<1, \lambda=1$, and $1<\lambda<2$. This is due to the different orders of growth of the sequence $\left(H_{\lambda}\left(\alpha_{N, \lambda}\right)-N^{2} I_{\lambda}\left(\sigma_{1}\right)\right)$.

Following the strategy employed in [13, we introduce the following definitions.

Definition 3.13. For a fixed $p \in \mathbb{N}$, let $\Theta_{p}$ denote the set of all vectors $\vec{\theta}=\left(\theta_{1}, \ldots, \theta_{p}\right)$ for which there exists an infinite sequence $\mathcal{N}$ of integers $N=2^{n_{1}}+\cdots+2^{n_{p}}, n_{1}>n_{2}>\cdots>n_{p} \geq 0$, satisfying

$$
\lim _{N \in \mathcal{N}} \frac{2^{n_{i}}}{N}=\theta_{i}, \quad \text { for all } i=1, \ldots, p .
$$




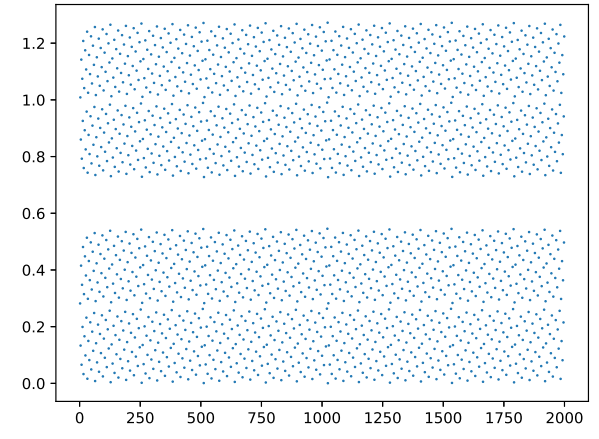

(a) $\lambda=1$

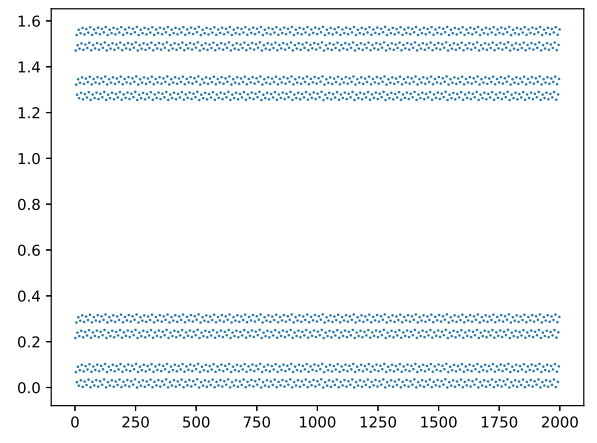

(c) $\lambda=1.5$

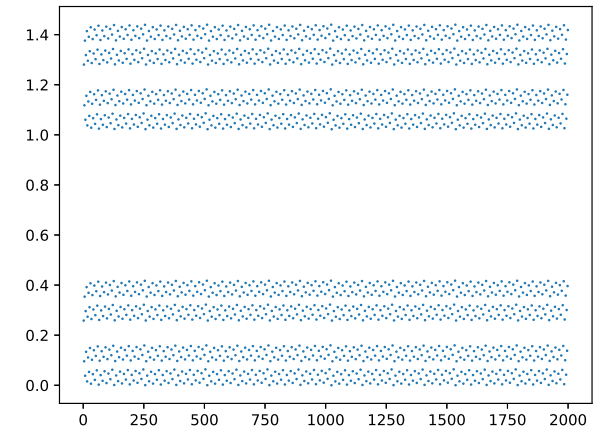

(b) $\lambda=1.3$

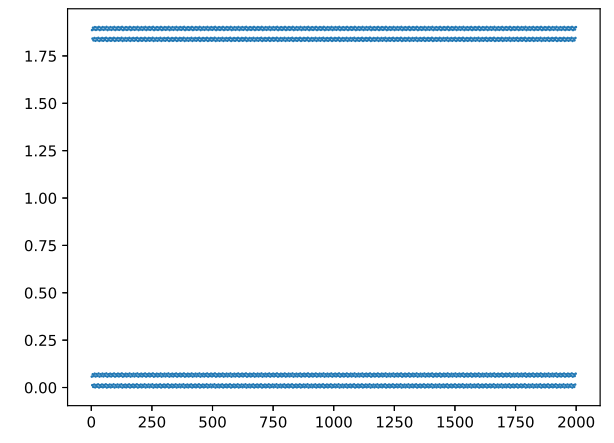

(d) $\lambda=1.9$

Figure 2: Plots of sequences $\left(U_{N}\left(a_{N}\right)-N I_{\lambda}\left(\sigma_{1}\right)\right)$ for $1 \leq \lambda<2$ and $1 \leq N \leq 2000$. 
Note that $\Theta_{p} \subset[0,1]^{p}$. If $\left(\theta_{1}, \ldots, \theta_{p}\right) \in \Theta_{p}$, then

$$
\sum_{i=1}^{p} \theta_{i}=1
$$

and $\theta_{i} \geq 2 \theta_{i+1}$ for each $1 \leq i \leq p-1$. In particular, $\theta_{1}>0$, and if $\theta_{i}=0$ for some $2 \leq i \leq p$, then $\theta_{j}=0$ for all $i \leq j \leq p$.

The asymptotic condition (3.36) is the defining property of $\Theta_{p}$ that we will apply, but this set can be defined alternatively as follows. $\Theta_{p}$ consists of all vectors $\vec{\theta}=\left(\theta_{1}, \ldots, \theta_{p}\right)$ that can be written in the form

$$
\vec{\theta}=\left(\frac{2^{t_{1}}}{M}, \frac{2^{t_{2}}}{M}, \ldots, \frac{2^{t_{r-1}}}{M}, \frac{1}{M}, 0, \ldots, 0\right),
$$

where $M=2^{t_{1}}+\cdots+2^{t_{r-1}}+1$ is an odd integer with $t_{1}>t_{2}>\cdots>t_{r-1}>0$ and $1 \leq r \leq p$. The number of zeros that appear in $\vec{\theta}$, if any, is $p-r$.

Definition 3.14. On $\Theta_{p} \times[0,1)$ we define the function

$$
G\left(\left(\theta_{1}, \ldots, \theta_{p}\right) ; \lambda\right):=\sum_{k=1}^{p} \theta_{k}^{-\lambda}\left(2\left(2^{-\lambda}-1\right)\left(\sum_{j=k+1}^{p} \theta_{j}\right)+\theta_{k}\right) .
$$

The inner sum is understood to be zero if $k=p$. Also, if $\theta_{k}=0$ for some $2 \leq k \leq p$, then the expression $\theta_{k}^{-\lambda}\left(2\left(2^{-\lambda}-1\right)\left(\sum_{j=k+1}^{p} \theta_{j}\right)+\theta_{k}\right)$ is understood to be zero, i.e.,

$$
\theta_{k}=0 \Longrightarrow \theta_{k}^{-\lambda}\left(2\left(2^{-\lambda}-1\right)\left(\sum_{j=k+1}^{p} \theta_{j}\right)+\theta_{k}\right)=0 .
$$

Observe that it is natural to adopt the convention (3.38) because $0 \leq \lambda<1$, which implies $\lim _{\theta \rightarrow 0^{+}} \theta^{1-\lambda}=0$.

Another important observation is that for any $\vec{\theta} \in \Theta_{p}$, we have $G(\vec{\theta} ; \lambda)>0$. Indeed, from the inequalities $1-\lambda>0$ and $\theta_{i} \geq 2 \theta_{i+1}, 1 \leq i \leq p-1$, it easily follows that for each $k$ with $\theta_{k}>0$, we have

$$
2\left(2^{-\lambda}-1\right)\left(\sum_{j=k+1}^{p} \theta_{j}\right)+\theta_{k}>0 .
$$

As a function of $\lambda, G(\vec{\theta} ; \lambda)$ is continuous, and $G(\vec{\theta} ; 0)=\sum_{k=1}^{p} \theta_{k}=1$.

Definition 3.15. Let $0 \leq \lambda<1$ be fixed. Using the function (3.37), we define

$$
\begin{aligned}
\bar{g}_{p}(\lambda) & :=\sup _{\vec{\theta} \in \Theta_{p}} G(\vec{\theta} ; \lambda), \quad p \in \mathbb{N}, \\
\bar{g}(\lambda) & :=\sup _{p \in \mathbb{N}} \bar{g}_{p}(\lambda) .
\end{aligned}
$$

Taking $\left(\theta_{1}, \theta_{2}\right)=(2 / 3,1 / 3) \in \Theta_{2}$, we have

$$
G\left(\left(\theta_{1}, \theta_{2}\right) ; \lambda\right)=\frac{2^{1-2 \lambda}+1}{3^{1-\lambda}}>1, \quad 0<\lambda<1,
$$




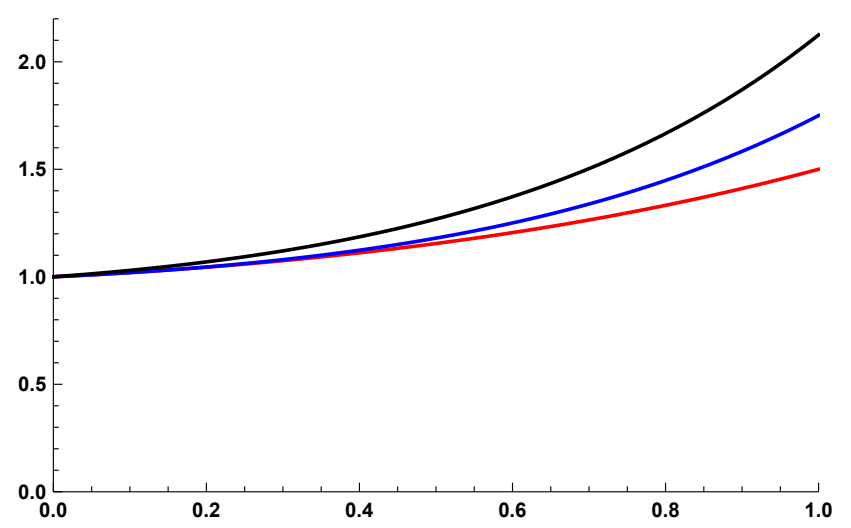

Figure 3: The figure shows the graphs of the functions (3.37) associated with the vectors $\vec{\theta}=$ $(2 / 3,1 / 3), \vec{\theta}=(4 / 7,2 / 7,1 / 7)$, and $\vec{\theta}=(8 / 11,2 / 11,1 / 11)$, in increasing order.

see also Figure 3, Hence

$$
\bar{g}(\lambda)>1, \quad 0<\lambda<1 .
$$

A key identity we will use is the following, which the reader can easily check. If $N$ has the binary representation (3.24), then

$$
N^{2}=\sum_{k=1}^{p-1}\left(\sum_{j=k+1}^{p} 2^{n_{j}-n_{k}}\right) 2^{2\left(n_{k}+1\right)}+\sum_{k=1}^{p}\left(1-\sum_{j=k+1}^{p} 2^{n_{j}-n_{k}+1}\right) 2^{2 n_{k}} .
$$

Note the similarity with the expression (3.25). We will also use the notation

$$
\mathcal{R}_{\lambda}(N):=\frac{\mathcal{L}_{\lambda}(N)-N^{2} I_{\lambda}\left(\sigma_{1}\right)}{N^{1-\lambda}}, \quad N \geq 1 .
$$

Then, according to (3.31), we have

$$
\lim _{N \rightarrow \infty} \mathcal{R}_{\lambda}(N)=(2 \pi)^{\lambda} 2 \zeta(-\lambda), \quad 0<\lambda<2 .
$$

It should also be noted that

$$
\mathcal{R}_{\lambda}(N)<0, \quad \text { for all } N \geq 1 .
$$

This follows from (3.23) and the second inequality in (3.8).

Given an integer $N \geq 1$, let $\tau(N)$ denote the number of ones in the binary digit representation of $N$, i.e., for an integer $N$ as in (3.24) we write $\tau(N)=p$.

The following result describes the second-order asymptotics of $H_{\lambda}\left(\alpha_{N, \lambda}\right)$ in the range $0<$ $\lambda<1$.

Theorem 3.16. Assume $0<\lambda<1$, let $\left(a_{n}\right)_{n=0}^{\infty} \subset S^{1}$ be a greedy $\lambda$-energy sequence, and $\alpha_{N, \lambda}$, $N \geq 2$, be the configuration (1.4). The sequence

$$
\left(\frac{H_{\lambda}\left(\alpha_{N, \lambda}\right)-N^{2} I_{\lambda}\left(\sigma_{1}\right)}{N^{1-\lambda}}\right)_{N=2}^{\infty}
$$


is bounded and divergent, and we have

$$
\begin{aligned}
& \limsup _{N \rightarrow \infty} \frac{H_{\lambda}\left(\alpha_{N, \lambda}\right)-N^{2} I_{\lambda}\left(\sigma_{1}\right)}{N^{1-\lambda}}=(2 \pi)^{\lambda} 2 \zeta(-\lambda), \\
& \liminf _{N \rightarrow \infty} \frac{H_{\lambda}\left(\alpha_{N, \lambda}\right)-N^{2} I_{\lambda}\left(\sigma_{1}\right)}{N^{1-\lambda}}=\bar{g}(\lambda)(2 \pi)^{\lambda} 2 \zeta(-\lambda) .
\end{aligned}
$$

For any $p \geq 1$ and $\vec{\theta} \in \Theta_{p}$, the value $G(\vec{\theta} ; \lambda)(2 \pi)^{\lambda} 2 \zeta(-\lambda)$ is a limit point ${ }^{\S}$ of the sequence (3.44). Proof. Let $N \geq 2$ be an integer with binary representation (3.24). Applying (3.25), (3.40), and (3.41), we get

$$
\begin{aligned}
\frac{H_{\lambda}\left(\alpha_{N, \lambda}\right)-N^{2} I_{\lambda}\left(\sigma_{1}\right)}{N^{1-\lambda}} & =\sum_{k=1}^{p-1}\left(\sum_{j=k+1}^{p} 2^{n_{j}-n_{k}}\right) \frac{\mathcal{L}_{\lambda}\left(2^{n_{k}+1}\right)-2^{2\left(n_{k}+1\right)} I_{\lambda}\left(\sigma_{1}\right)}{N^{1-\lambda}} \\
& +\sum_{k=1}^{p}\left(1-\sum_{j=k+1}^{p} 2^{n_{j}-n_{k}+1}\right) \frac{\mathcal{L}_{\lambda}\left(2^{n_{k}}\right)-2^{2 n_{k}} I_{\lambda}\left(\sigma_{1}\right)}{N^{1-\lambda}} \\
& =\sum_{k=1}^{p-1}\left(\sum_{j=k+1}^{p} 2^{n_{j}-n_{k}}\right)\left(\frac{2^{n_{k}+1}}{N}\right)^{1-\lambda} \mathcal{R}_{\lambda}\left(2^{n_{k}+1}\right) \\
& +\sum_{k=1}^{p}\left(1-\sum_{j=k+1}^{p} 2^{n_{j}-n_{k}+1}\right)\left(\frac{2^{n_{k}}}{N}\right)^{1-\lambda} \mathcal{R}_{\lambda}\left(2^{n_{k}}\right) .
\end{aligned}
$$

First we show that the sequence (3.44) is bounded. The sequence (3.41) converges, so there exists $C>0$ such that $\left|\mathcal{R}_{\lambda}(N)\right|<C$ for all $N \geq 1$. Therefore, applying the triangle inequality in (3.47),

$$
\leq C\left(\sum_{k=1}^{p-1}\left(\sum_{j=k+1}^{p} 2^{n_{j}-n_{k}}\right)\left(\frac{\left.\left.{\frac{H_{\lambda}}{n_{k}+1}}_{N}\right)^{1-\lambda}\right)-N^{2} I_{\lambda}\left(\sigma_{1}\right)}{N^{1-\lambda}} \mid \sum_{k=1}^{p}\left(1+\sum_{j=k+1}^{p} 2^{n_{j}-n_{k}+1}\right)\left(\frac{2^{n_{k}}}{N}\right)^{1-\lambda}\right) .\right.
$$

Now, we have $n_{j}-n_{k} \leq-(j-k)$ for every $k+1 \leq j \leq p$, hence

$$
\sum_{j=k+1}^{p} 2^{n_{j}-n_{k}} \leq 2^{-1}+2^{-2}+\cdots+2^{-(p-k)}<1 .
$$

Similarly,

So we have the estimate

$$
\left(\frac{2^{n_{k}}}{N}\right)^{1-\lambda} \leq\left(\frac{2^{n_{k}}}{2^{n_{1}}}\right)^{1-\lambda} \leq 2^{-(1-\lambda)(k-1)}
$$

$$
\left|\frac{H_{\lambda}\left(\alpha_{N, \lambda}\right)-N^{2} I_{\lambda}\left(\sigma_{1}\right)}{N^{1-\lambda}}\right| \leq C\left(2^{1-\lambda}+3\right) \sum_{k=1}^{p} 2^{-(1-\lambda)(k-1)}<C \frac{2^{1-\lambda}+3}{1-2^{\lambda-1}}<\infty .
$$

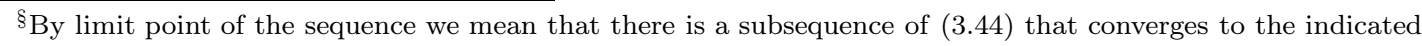
value.
} 
This justifies the boundedness of (3.44).

For every $N \geq 2$, we have $H_{\lambda}\left(\alpha_{N, \lambda}\right) \leq \mathcal{L}_{\lambda}(N)$ because $\mathcal{L}_{\lambda}(N)$ is the $\lambda$-energy of a configuration of $N$ equally spaced points on $S^{1}$, which is the largest among all $N$-point configurations on the circle. Since $\alpha_{2^{n}, \lambda}$ is the configuration of the $2^{n}$-th roots of unity, we have $H_{\lambda}\left(\alpha_{N, \lambda}\right)=\mathcal{L}_{\lambda}(N)$ for each $N=2^{n}$, hence

$$
\limsup _{N \rightarrow \infty} \frac{H_{\lambda}\left(\alpha_{N, \lambda}\right)-N^{2} I_{\lambda}\left(\sigma_{1}\right)}{N^{1-\lambda}}=\lim _{n \rightarrow \infty} \mathcal{R}_{\lambda}\left(2^{n}\right)=(2 \pi)^{\lambda} 2 \zeta(-\lambda) .
$$

This is 3.45).

Now we turn to the proof of (3.46). Fix $p \geq 1$, and let $\vec{\theta}=\left(\theta_{1}, \ldots, \theta_{p}\right) \in \Theta_{p}$. By definition, there exists an infinite sequence $\mathcal{N} \subset \mathbb{N}$ such that each $N \in \mathcal{N}$ has a binary representation (3.24) of length $p$, and (3.36) holds. Fix $1 \leq k \leq p$, and assume first that the corresponding $\theta_{k}$ is not zero, i.e.,

$$
\lim _{N \in \mathcal{N}} \frac{2^{n_{k}}}{N}=\theta_{k}>0
$$

In this case, we clearly have $2^{n_{k}} \rightarrow \infty$ as $N \in \mathcal{N}$ approaches infinity. Therefore, in virtue of (3.36) and (3.42), we have

$$
\begin{aligned}
\lim _{N \in \mathcal{N}} \mathcal{R}_{\lambda}\left(2^{n_{k}}\right) & =\lim _{N \in \mathcal{N}} \mathcal{R}_{\lambda}\left(2^{n_{k}+1}\right)=(2 \pi)^{\lambda} 2 \zeta(-\lambda) \\
\lim _{N \in \mathcal{N}}\left(\frac{2^{n_{k}+1}}{N}\right)^{1-\lambda} & =\left(2 \theta_{k}\right)^{1-\lambda} \quad \lim _{N \in \mathcal{N}}\left(\frac{2^{n_{k}}}{N}\right)^{1-\lambda}=\theta_{k}^{1-\lambda} \\
\lim _{N \in \mathcal{N}} 2^{n_{j}-n_{k}} & =\frac{\theta_{j}}{\theta_{k}} \quad \text { for each } k+1 \leq j \leq p .
\end{aligned}
$$

Assume now that the index $k$ is such that

$$
\lim _{N \in \mathcal{N}} \frac{2^{n_{k}}}{N}=\theta_{k}=0
$$

In this case, since $0 \leq \lambda<1$, the sequence $\left(\mathcal{R}_{\lambda}(N)\right)$ is bounded, and we have (3.48), it follows that

$$
\begin{aligned}
& \lim _{N \in \mathcal{N}}\left(\sum_{j=k+1}^{p} 2^{n_{j}-n_{k}}\right)\left(\frac{2^{n_{k}+1}}{N}\right)^{1-\lambda} \mathcal{R}_{\lambda}\left(2^{n_{k}+1}\right)=0, \\
& \lim _{N \in \mathcal{N}}\left(1-\sum_{j=k+1}^{p} 2^{n_{j}-n_{k}+1}\right)\left(\frac{2^{n_{k}}}{N}\right)^{1-\lambda} \mathcal{R}_{\lambda}\left(2^{n_{k}}\right)=0 .
\end{aligned}
$$

Suppose that $1 \leq \widehat{\kappa} \leq p$ is the last component of $\vec{\theta}=\left(\theta_{1}, \ldots, \theta_{p}\right)$ that is non-zero, i.e.,

$$
\begin{array}{ll}
\theta_{i}>0 & \text { for } 1 \leq i \leq \widehat{\kappa}, \\
\theta_{i}=0 & \text { for } \widehat{\kappa}+1 \leq i \leq p .
\end{array}
$$

Then, applying (3.47), (3.50), and (3.51), we obtain

$$
\begin{aligned}
\lim _{N \in \mathcal{N}} \frac{H_{\lambda}\left(\alpha_{N, \lambda}\right)-N^{2} I_{\lambda}\left(\sigma_{1}\right)}{N^{1-\lambda}} & =\left(\sum_{k=1}^{\widehat{\kappa}} \theta_{k}^{-\lambda}\left(2\left(2^{-\lambda}-1\right)\left(\sum_{j=k+1}^{\widehat{\kappa}} \theta_{j}\right)+\theta_{k}\right)\right)(2 \pi)^{\lambda} 2 \zeta(-\lambda) \\
& =G\left(\left(\theta_{1}, \ldots, \theta_{p}\right) ; \lambda\right)(2 \pi)^{\lambda} 2 \zeta(-\lambda),
\end{aligned}
$$


where in the second equality we have used (3.38). This shows that $G\left(\left(\theta_{1}, \ldots, \theta_{p}\right) ; \lambda\right)(2 \pi)^{\lambda} 2 \zeta(-\lambda)$ is a limit point of the sequence (3.44). Since $p$ and $\vec{\theta}=\left(\theta_{1}, \ldots, \theta_{p}\right)$ were arbitrary, this implies

$$
\liminf _{N \rightarrow \infty} \frac{H_{\lambda}\left(\alpha_{N, \lambda}\right)-N^{2} I_{\lambda}\left(\sigma_{1}\right)}{N^{1-\lambda}} \leq \bar{g}(\lambda)(2 \pi)^{\lambda} 2 \zeta(-\lambda),
$$

recall that $\zeta(-\lambda)<0$ for $\lambda \in(0,1)$.

To finish, we need to justify the inequality

$$
\liminf _{N \rightarrow \infty} \frac{H_{\lambda}\left(\alpha_{N, \lambda}\right)-N^{2} I_{\lambda}\left(\sigma_{1}\right)}{N^{1-\lambda}} \geq \bar{g}(\lambda)(2 \pi)^{\lambda} 2 \zeta(-\lambda) .
$$

Let $\mathcal{N} \subset \mathbb{N}$ be an infinite sequence for which the sequence $\left(\frac{H_{\lambda}\left(\alpha_{N, \lambda}\right)-N^{2} I_{\lambda}\left(\sigma_{1}\right)}{N^{1-\lambda}}\right)_{N \in \mathcal{N}}$ converges, and let us show that

$$
\lim _{N \in \mathcal{N}} \frac{H_{\lambda}\left(\alpha_{N, \lambda}\right)-N^{2} I_{\lambda}\left(\sigma_{1}\right)}{N^{1-\lambda}} \geq \bar{g}(\lambda)(2 \pi)^{\lambda} 2 \zeta(-\lambda) .
$$

First assume that there exists $p \geq 1$ such that infinitely many $N \in \mathcal{N}$ satisfy $\tau(N)=p$. Then, passing to a subsequence $\tilde{\mathcal{N}}$ of $\mathcal{N}$ if necessary, we may assume that the integers $N=$ $2^{n_{1}}+\cdots+2^{n_{p}} \in \widetilde{\mathcal{N}}$ satisfy

$$
\lim _{N \in \widetilde{\mathcal{N}}} \frac{2^{n_{i}}}{N}=\theta_{i}, \quad \text { for all } i=1, \ldots, p .
$$

In this situation, we have already shown that we get

$$
\lim _{N \in \mathcal{N}} \frac{H_{\lambda}\left(\alpha_{N, \lambda}\right)-N^{2} I_{\lambda}\left(\sigma_{1}\right)}{N^{1-\lambda}}=\lim _{N \in \tilde{\mathcal{N}}} \frac{H_{\lambda}\left(\alpha_{N, \lambda}\right)-N^{2} I_{\lambda}\left(\sigma_{1}\right)}{N^{1-\lambda}}=G\left(\left(\theta_{1}, \ldots, \theta_{p}\right) ; \lambda\right)(2 \pi)^{\lambda} 2 \zeta(-\lambda),
$$

and (3.52) holds.

Assume now that such an integer $p$ does not exist. This means that we have $\tau(N) \rightarrow \infty$ as $N \rightarrow \infty$ along the sequence $\mathcal{N}$. For $N=2^{n_{1}}+\cdots+2^{n_{\tau(N)}} \in \mathcal{N}, n_{1}>n_{2}>\cdots>n_{\tau(N)} \geq 0$, we rewrite (3.47):

$$
\begin{gathered}
\frac{H_{\lambda}\left(\alpha_{N, \lambda}\right)-N^{2} I_{\lambda}\left(\sigma_{1}\right)}{N^{1-\lambda}} \\
=\sum_{k=1}^{\tau(N)}\left(\frac{2^{n_{k}}}{N}\right)^{1-\lambda}\left(2^{1-\lambda} \mathcal{R}_{\lambda}\left(2^{n_{k}+1}\right) \sum_{j=k+1}^{\tau(N)} 2^{n_{j}-n_{k}}+\mathcal{R}_{\lambda}\left(2^{n_{k}}\right)\left(1-\sum_{j=k+1}^{\tau(N)} 2^{n_{j}-n_{k}+1}\right)\right) .
\end{gathered}
$$

The idea is now to truncate adequately the sums that appear in (3.53). Let us define

$$
\begin{aligned}
\eta_{N, k} & :=2^{1-\lambda} \mathcal{R}_{\lambda}\left(2^{n_{k}+1}\right) \sum_{j=k+1}^{\tau(N)} 2^{n_{j}-n_{k}}+\mathcal{R}_{\lambda}\left(2^{n_{k}}\right)\left(1-\sum_{j=k+1}^{\tau(N)} 2^{n_{j}-n_{k}+1}\right) \\
& =\mathcal{R}_{\lambda}\left(2^{n_{k}}\right)+\left(2^{-\lambda} \mathcal{R}_{\lambda}\left(2^{n_{k}+1}\right)-\mathcal{R}_{\lambda}\left(2^{n_{k}}\right)\right) \sum_{j=k+1}^{\tau(N)} 2^{n_{j}-n_{k}+1} .
\end{aligned}
$$

The boundedness of the sequence $\left(\mathcal{R}_{\lambda}(N)\right)$ and (3.48) imply that there exists an absolute constant $C_{1}>0$ such that

$$
\left|\eta_{N, k}\right| \leq C_{1}, \quad \text { for all } N \in \mathcal{N} \text { and } 1 \leq k \leq \tau(N) .
$$


Let $0<\epsilon<1$ be fixed. Let $M=M(\epsilon, \lambda) \geq 1$ be large enough so that

$$
\sum_{k=M}^{\infty} 2^{-(1-\lambda) k}<\epsilon
$$

The following choice suffices:

$$
M:=\left\lceil-\frac{\log \left(\epsilon\left(2^{1-\lambda}-1\right)\right)}{(1-\lambda) \log 2}\right\rceil+2,
$$

where $\lceil\cdot\rceil$ is the ceiling function.

We can write

$$
\frac{H_{\lambda}\left(\alpha_{N, \lambda}\right)-N^{2} I_{\lambda}\left(\sigma_{1}\right)}{N^{1-\lambda}}=S_{N, M, 1}+S_{N, M, 2}
$$

where

$$
S_{N, M, 1}:=\sum_{k=1}^{M}\left(\frac{2^{n_{k}}}{N}\right)^{1-\lambda} \eta_{N, k}, \quad S_{N, M, 2}:=\sum_{k=M+1}^{\tau(N)}\left(\frac{2^{n_{k}}}{N}\right)^{1-\lambda} \eta_{N, k} .
$$

Then, from (3.49), (3.55), and (3.56), we deduce

$$
\left|S_{N, M, 2}\right| \leq C_{1} \epsilon, \quad \text { for all } N \in \mathcal{N} .
$$

Now we concentrate our analysis on $S_{N, M, 1}$. So in the rest of the proof, $k$ is an index in the range $1 \leq k \leq M$.

We split the expression of $\eta_{N, k}$ in (3.54) as follows:

$$
\eta_{N, k}=\eta_{N, k, 1}+\eta_{N, k, 2}
$$

where

$$
\begin{aligned}
& \eta_{N, k, 1}:=\mathcal{R}_{\lambda}\left(2^{n_{k}}\right)+\left(2^{-\lambda} \mathcal{R}_{\lambda}\left(2^{n_{k}+1}\right)-\mathcal{R}_{\lambda}\left(2^{n_{k}}\right)\right) \sum_{j=k+1}^{2 M} 2^{n_{j}-n_{k}+1}, \\
& \eta_{N, k, 2}:=\left(2^{-\lambda} \mathcal{R}_{\lambda}\left(2^{n_{k}+1}\right)-\mathcal{R}_{\lambda}\left(2^{n_{k}}\right)\right) \sum_{j=2 M+1}^{\tau(N)} 2^{n_{j}-n_{k}+1}
\end{aligned}
$$

Recall that $n_{j}-n_{k} \leq-(j-k)$ for all $j \geq k+1$, hence if $1 \leq k \leq M$ and $2 M+1 \leq j$, then

$$
\sum_{j=2 M+1}^{\tau(N)} 2^{n_{j}-n_{k}+1} \leq \sum_{j=2 M+1}^{\tau(N)} 2^{-j+M+1}<\sum_{t=M}^{\infty} 2^{-t}<\epsilon
$$

where we used (3.56). So by the boundedness of the sequence $\left(\mathcal{R}_{\lambda}(N)\right)$, we obtain that there exists an absolute constant $C_{2}>0$ such that

$$
\left|\eta_{N, k, 2}\right|<C_{2} \epsilon, \quad \text { for all } N \in \mathcal{N} \text { and } 1 \leq k \leq M .
$$

Now, by definition of $S_{N, M, 1}$ and (3.60), we have

$$
S_{N, M, 1}=\sum_{k=1}^{M}\left(\frac{2^{n_{k}}}{N}\right)^{1-\lambda} \eta_{N, k, 1}+\sum_{k=1}^{M}\left(\frac{2^{n_{k}}}{N}\right)^{1-\lambda} \eta_{N, k, 2} .
$$


From the estimate (3.61) we get

$$
\left|\sum_{k=1}^{M}\left(\frac{2^{n_{k}}}{N}\right)^{1-\lambda} \eta_{N, k, 2}\right| \leq C_{2} M \epsilon .
$$

We apply (3.58), (3.59), and (3.62), and conclude that

$$
\begin{gathered}
\frac{H_{\lambda}\left(\alpha_{N, \lambda}\right)-N^{2} I_{\lambda}\left(\sigma_{1}\right)}{N^{1-\lambda}}=\phi_{N}+\sum_{k=1}^{M}\left(\frac{2^{n_{k}}}{N}\right)^{1-\lambda} \eta_{N, k, 1} \\
=\phi_{N}+\sum_{k=1}^{M}\left(\frac{2^{n_{k}}}{N}\right)^{1-\lambda}\left(\mathcal{R}_{\lambda}\left(2^{n_{k}}\right)+\left(2^{-\lambda} \mathcal{R}_{\lambda}\left(2^{n_{k}+1}\right)-\mathcal{R}_{\lambda}\left(2^{n_{k}}\right)\right) \sum_{j=k+1}^{2 M} 2^{n_{j}-n_{k}+1}\right)
\end{gathered}
$$

where

$$
\left|\phi_{N}\right| \leq C_{1} \epsilon+C_{2} M \epsilon, \quad \text { for all } N \in \mathcal{N}
$$

Since $M$ is a fixed finite constant, there exists a subsequence $\widetilde{\mathcal{N}} \subset \mathcal{N}$ such that

$$
\lim _{N \in \widetilde{\mathcal{N}}} \frac{2^{n_{i}}}{N}=\theta_{i}, \quad \text { for all } 1 \leq i \leq 2 M .
$$

If $\theta_{k}>0$ for some $1 \leq k \leq M$, then $2^{n_{k}} \rightarrow \infty$ as $N \in \widetilde{\mathcal{N}}$ tends to infinity, hence it follows from (3.42) and (3.65) that for this $k$,

$$
\begin{gathered}
\lim _{N \in \widetilde{\mathcal{N}}}\left(\frac{2^{n_{k}}}{N}\right)^{1-\lambda}\left(\mathcal{R}_{\lambda}\left(2^{n_{k}}\right)+\left(2^{-\lambda} \mathcal{R}_{\lambda}\left(2^{n_{k}+1}\right)-\mathcal{R}_{\lambda}\left(2^{n_{k}}\right)\right) \sum_{j=k+1}^{2 M} 2^{n_{j}-n_{k}+1}\right) \\
=\theta_{k}^{-\lambda}\left(2\left(2^{-\lambda}-1\right)\left(\sum_{j=k+1}^{2 M} \theta_{j}\right)+\theta_{k}\right)(2 \pi)^{\lambda} 2 \zeta(-\lambda) .
\end{gathered}
$$

And if $\theta_{k}=0$ for some $1 \leq k \leq M$, then as explained before (see (3.51)), the limit in (3.66) is zero. In this case we can also express the limit value zero as the right-hand side of (3.66), keeping in mind the convention (3.38).

From (3.63) and (3.66) we obtain

$$
\begin{aligned}
\lim _{N \in \mathcal{N}} \frac{H_{\lambda}\left(\alpha_{N, \lambda}\right)-N^{2} I_{\lambda}\left(\sigma_{1}\right)}{N^{1-\lambda}} & =\lim _{N \in \widetilde{\mathcal{N}}} \frac{H_{\lambda}\left(\alpha_{N, \lambda}\right)-N^{2} I_{\lambda}\left(\sigma_{1}\right)}{N^{1-\lambda}} \\
& =\lim _{N \in \widetilde{\mathcal{N}}} \phi_{N}+\lim _{N \in \widetilde{\mathcal{N}}} \sum_{k=1}^{M}\left(\frac{2^{n_{k}}}{N}\right)^{1-\lambda} \eta_{N, k, 1} \\
& =\lim _{N \in \widetilde{\mathcal{N}}} \phi_{N}+(2 \pi)^{\lambda} 2 \zeta(-\lambda)\left(\sum_{k=1}^{M} \theta_{k}^{-\lambda}\left(2\left(2^{-\lambda}-1\right)\left(\sum_{j=k+1}^{2 M} \theta_{j}\right)+\theta_{k}\right)\right) \\
& \geq \lim _{N \in \widetilde{\mathcal{N}}} \phi_{N}+(2 \pi)^{\lambda} 2 \zeta(-\lambda)\left(\sum_{k=1}^{M} \theta_{k}^{-\lambda}\left(2\left(2^{-\lambda}-1\right)\left(\sum_{j=k+1}^{M} \theta_{j}\right)+\theta_{k}\right)\right) \\
& =\lim _{N \in \widetilde{\mathcal{N}}} \phi_{N}+(2 \pi)^{\lambda} 2 \zeta(-\lambda) G\left(\left(\theta_{1}, \ldots, \theta_{M}\right) ; \lambda\right) \\
& \geq \lim _{N \in \widetilde{\mathcal{N}}} \phi_{N}+\bar{g}(\lambda)(2 \pi)^{\lambda} 2 \zeta(-\lambda) \\
& \geq-\left(C_{1} \epsilon+C_{2} M(\epsilon) \epsilon\right)+\bar{g}(\lambda)(2 \pi)^{\lambda} 2 \zeta(-\lambda)
\end{aligned}
$$


where we used (3.64) in the last inequality. Letting $\epsilon \rightarrow 0$, we get $M(\epsilon) \epsilon \rightarrow 0$ (see (3.57)), and (3.52) is proved. This finishes the proof of (3.46).

The estimate (3.39) implies that the limit values in (3.45) and (3.46) are different, so the sequence (3.44) diverges.

In Figure 4 we present plots of the normalized second-order sequences (3.44), for different values of $\lambda \in(0,1)$.

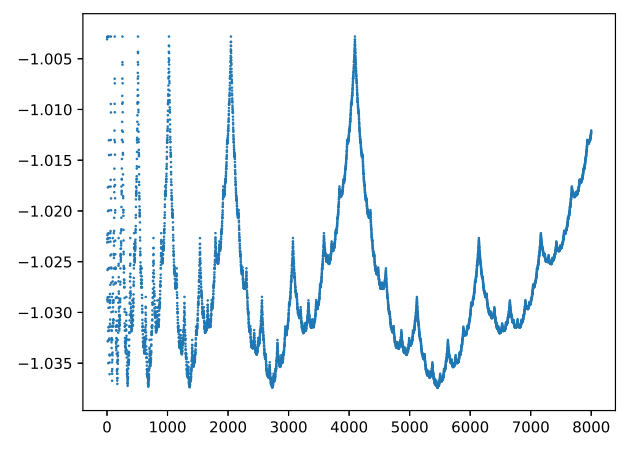

(a) $\lambda=0.1$

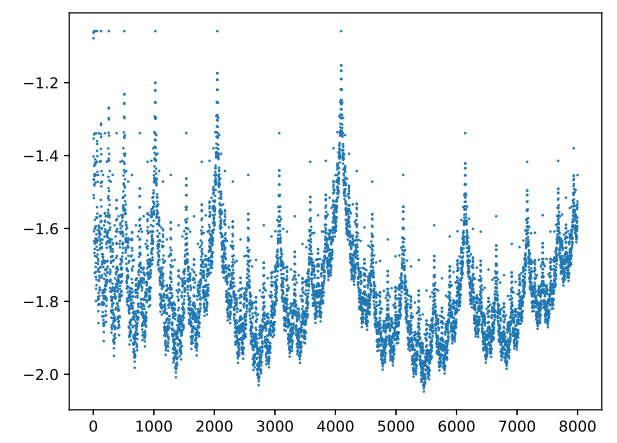

(c) $\lambda=0.7$

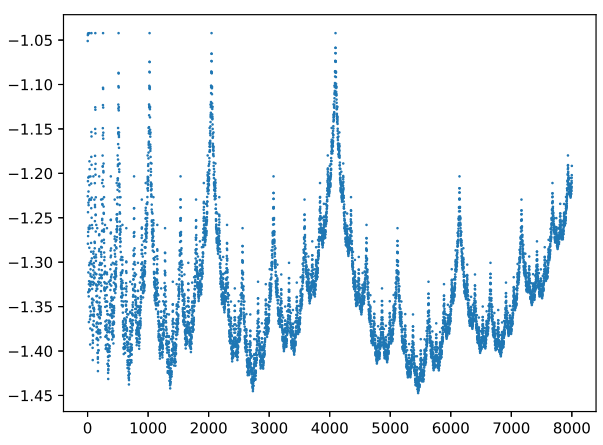

(b) $\lambda=0.5$

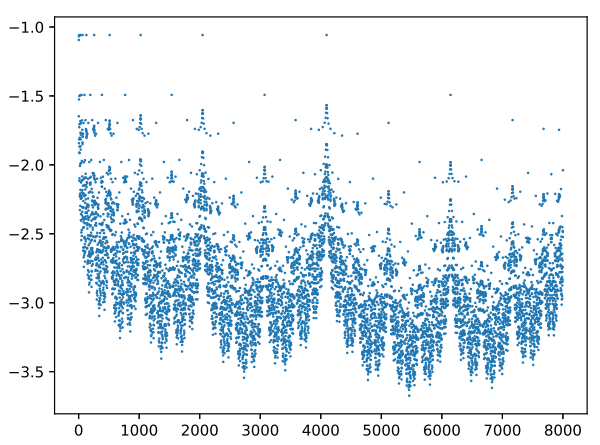

(d) $\lambda=0.9$

Figure 4: Plots of the normalized second-order energy (3.44) for $0<\lambda<1$ and $2 \leq N \leq 8000$.

The next result concerns the second-order asymptotics of $H_{\lambda}\left(\alpha_{N, \lambda}\right)$ in the range $1<\lambda<2$.

Theorem 3.17. Assume $1<\lambda<2$, let $\left(a_{n}\right)_{n=0}^{\infty} \subset S^{1}$ be a greedy $\lambda$-energy sequence, and $\alpha_{N, \lambda}$, $N \geq 2$, be the configuration (1.4). The sequence

$$
\left(H_{\lambda}\left(\alpha_{N, \lambda}\right)-N^{2} I_{\lambda}\left(\sigma_{1}\right)\right)_{N=2}^{\infty}
$$

is bounded and divergent. We have

$$
\begin{aligned}
& \limsup _{N \rightarrow \infty}\left(H_{\lambda}\left(\alpha_{N, \lambda}\right)-N^{2} I_{\lambda}\left(\sigma_{1}\right)\right)=0 \\
& \liminf _{N \rightarrow \infty}\left(H_{\lambda}\left(\alpha_{N, \lambda}\right)-N^{2} I_{\lambda}\left(\sigma_{1}\right)\right) \leq s_{\lambda}
\end{aligned}
$$


where ${ }^{\S}$

$$
s_{\lambda}:=\frac{1}{3} \sum_{k=0}^{\infty}\left(1+\frac{(-1)^{k}}{2^{k-1}}\right)\left(\mathcal{L}_{\lambda}\left(2^{k}\right)-2^{2 k} I_{\lambda}\left(\sigma_{1}\right)\right)
$$

We also have

$$
\liminf _{N \rightarrow \infty}\left(H_{\lambda}\left(\alpha_{N, \lambda}\right)-N^{2} I_{\lambda}\left(\sigma_{1}\right)\right)=\inf _{N \geq 2}\left\{H_{\lambda}\left(\alpha_{N, \lambda}\right)-N^{2} I_{\lambda}\left(\sigma_{1}\right)\right\} .
$$

Each term in the sequence (3.67) is a limit point of the sequence itself.

Proof. Let $N \geq 2$ be as in (3.24). Applying (3.25), (3.40), and (3.41), we have

$$
\begin{aligned}
H_{\lambda}\left(\alpha_{N, \lambda}\right)-N^{2} I_{\lambda}\left(\sigma_{1}\right) & =\sum_{k=1}^{p-1}\left(\sum_{j=k+1}^{p} 2^{n_{j}-n_{k}}\right)\left(2^{n_{k}+1}\right)^{1-\lambda} \mathcal{R}_{\lambda}\left(2^{n_{k}+1}\right) \\
& +\sum_{k=1}^{p}\left(1-\sum_{j=k+1}^{p} 2^{n_{j}-n_{k}+1}\right)\left(2^{n_{k}}\right)^{1-\lambda} \mathcal{R}_{\lambda}\left(2^{n_{k}}\right) .
\end{aligned}
$$

The sequence $\left(\mathcal{R}_{\lambda}(N)\right)$ is bounded, and let $C>0$ be a constant such that $\left|\mathcal{R}_{\lambda}(N)\right|<C$ for all $N$. Applying the triangle inequality in (3.72), we get

$$
\begin{gathered}
\left|H_{\lambda}\left(\alpha_{N, \lambda}\right)-N^{2} I_{\lambda}\left(\sigma_{1}\right)\right| \\
\leq C\left(\sum_{k=1}^{p-1}\left(\sum_{j=k+1}^{p} 2^{n_{j}-n_{k}}\right)\left(2^{n_{k}+1}\right)^{1-\lambda}+\sum_{k=1}^{p}\left(1+\sum_{j=k+1}^{p} 2^{n_{j}-n_{k}+1}\right)\left(2^{n_{k}}\right)^{1-\lambda}\right) .
\end{gathered}
$$

Using (3.48) and the estimate $\sum_{k=1}^{p}\left(2^{n_{k}}\right)^{1-\lambda}<\sum_{t=0}^{\infty}\left(2^{1-\lambda}\right)^{t}=1 /\left(1-2^{1-\lambda}\right)$, we obtain the uniform bound

$$
\left|H_{\lambda}\left(\alpha_{N, \lambda}\right)-N^{2} I_{\lambda}\left(\sigma_{1}\right)\right| \leq C \frac{2^{1-\lambda}+3}{1-2^{1-\lambda}}
$$

so the sequence (3.67) is bounded.

We have, for all $N \geq 2$,

$$
H_{\lambda}\left(\alpha_{N, \lambda}\right)-N^{2} I_{\lambda}\left(\sigma_{1}\right) \leq \mathcal{L}_{\lambda}(N)-N^{2} I_{\lambda}\left(\sigma_{1}\right)=N^{1-\lambda} \mathcal{R}_{\lambda}(N),
$$

with equality if $N=2^{n}, n \geq 1$. Since $\lim _{N \rightarrow \infty} N^{1-\lambda} \mathcal{R}_{\lambda}(N)=0$, we obtain (3.68).

Let us justify the identity (3.71) now. If we show that for each fixed $\widehat{N}=2^{n_{1}}+\cdots+2^{n_{p}}$, $n_{1}>\ldots>n_{p}$, we have

$$
\liminf _{N \rightarrow \infty}\left(H_{\lambda}\left(\alpha_{N, \lambda}\right)-N^{2} I_{\lambda}\left(\sigma_{1}\right)\right) \leq H_{\lambda}\left(\alpha_{\widehat{N}, \lambda}\right)-\widehat{N}^{2} I_{\lambda}\left(\sigma_{1}\right),
$$

then (3.71) will be justified. For such $\widehat{N}$ fixed, take $\widetilde{N}=2^{n_{0}}+\widehat{N}$, and let $n_{0} \rightarrow \infty$. Since $\left(2^{n_{0}}\right)^{1-\lambda} \rightarrow 0$, it easily follows from (3.72) that

$$
\lim _{n_{0} \rightarrow \infty}\left(H_{\lambda}\left(\alpha_{\widetilde{N}, \lambda}\right)-\widetilde{N}^{2} I_{\lambda}\left(\sigma_{1}\right)\right)=H_{\lambda}\left(\alpha_{\widehat{N}, \lambda}\right)-\widehat{N}^{2} I_{\lambda}\left(\sigma_{1}\right),
$$

which justifies (3.73), and shows that every term in the sequence (3.67) is a limit point of the sequence itself.

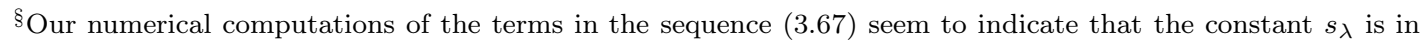
fact the liminf of the sequence 3.67.
} 
Now we justify (3.69). Consider the subsequence

$$
N(p):=\sum_{k=0}^{p-1} 2^{2 k}=\frac{4^{p}-1}{3} .
$$

Applying (3.72) for $N=N(p)$, we have

$$
\begin{aligned}
H_{\lambda}\left(\alpha_{N, \lambda}\right)-N^{2} I_{\lambda}\left(\sigma_{1}\right) & =\sum_{k=1}^{p-1} \frac{1}{3}\left(1-\frac{1}{4^{p-k}}\right)\left(2^{2(p-k)+1}\right)^{1-\lambda} \mathcal{R}_{\lambda}\left(2^{2(p-k)+1}\right) \\
& +\sum_{k=1}^{p} \frac{1}{3}\left(1+\frac{2}{4^{p-k}}\right)\left(2^{2(p-k)}\right)^{1-\lambda} \mathcal{R}_{\lambda}\left(2^{2(p-k)}\right) \\
& =\frac{1}{3} \sum_{m=0}^{p-1}\left(1-\frac{1}{4^{m}}\right)\left(\mathcal{L}_{\lambda}\left(2^{2 m+1}\right)-2^{4 m+2} I_{\lambda}\left(\sigma_{1}\right)\right) \\
& +\frac{1}{3} \sum_{m=0}^{p-1}\left(1+\frac{2}{4^{m}}\right)\left(\mathcal{L}_{\lambda}\left(2^{2 m}\right)-2^{4 m} I_{\lambda}\left(\sigma_{1}\right)\right)
\end{aligned}
$$

where in the second equality we applied the substitution $m=p-k$ and (3.41). This sequence is bounded and monotonic (every term in the sums is $\leq 0$ ), so it converges to

$$
\begin{aligned}
\lim _{p \rightarrow \infty}\left(H_{\lambda}\left(\alpha_{N, \lambda}\right)-N^{2} I_{\lambda}\left(\sigma_{1}\right)\right) & =\frac{1}{3} \sum_{m=0}^{\infty}\left(1-\frac{1}{4^{m}}\right)\left(\mathcal{L}_{\lambda}\left(2^{2 m+1}\right)-2^{4 m+2} I_{\lambda}\left(\sigma_{1}\right)\right) \\
& +\frac{1}{3} \sum_{m=0}^{\infty}\left(1+\frac{2}{4^{m}}\right)\left(\mathcal{L}_{\lambda}\left(2^{2 m}\right)-2^{4 m} I_{\lambda}\left(\sigma_{1}\right)\right)
\end{aligned}
$$

and this is exactly (3.70).

Each term in the series (3.70) is negative, except the second one, which is zero. The first term in the series is $-I_{\lambda}\left(\sigma_{1}\right)$, so $s_{\lambda}<-I_{\lambda}\left(\sigma_{1}\right)<0$. Hence, the sequence (3.67) is divergent.

In Figure 5 we present some plots of sequences (3.67) in the range $1<\lambda<2$.

Our next result concerns the second-order asymptotics of $H_{\lambda}\left(\alpha_{N, \lambda}\right)$ in the case $\lambda=1$.

Theorem 3.18. Let $\left(a_{n}\right)_{n=0}^{\infty} \subset S^{1}$ be a greedy 1-energy sequence, and let $\alpha_{N, 1}, N \geq 2$, be the configuration in (1.4). The sequence

$$
\left(\frac{H_{1}\left(\alpha_{N, 1}\right)-N^{2} I_{1}\left(\sigma_{1}\right)}{\log N}\right)_{N=2}^{\infty}
$$

is bounded and divergent. We have

$$
\begin{aligned}
& \limsup _{N \rightarrow \infty} \frac{H_{1}\left(\alpha_{N, 1}\right)-N^{2} I_{1}\left(\sigma_{1}\right)}{\log N}=0, \\
& \liminf _{N \rightarrow \infty} \frac{H_{1}\left(\alpha_{N, 1}\right)-N^{2} I_{1}\left(\sigma_{1}\right)}{\log N} \leq-\frac{\pi}{9 \log 2} .
\end{aligned}
$$




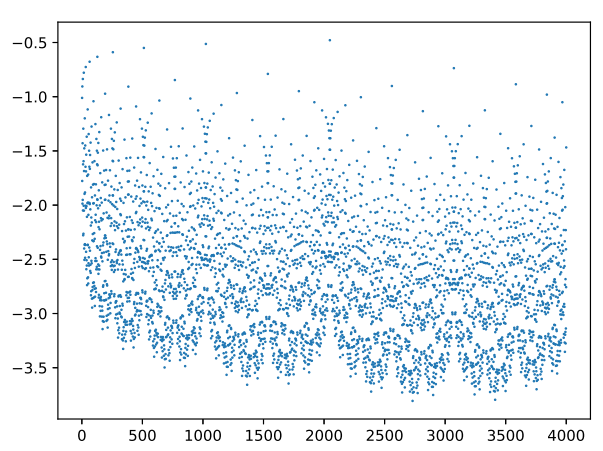

(a) $\lambda=1.1$

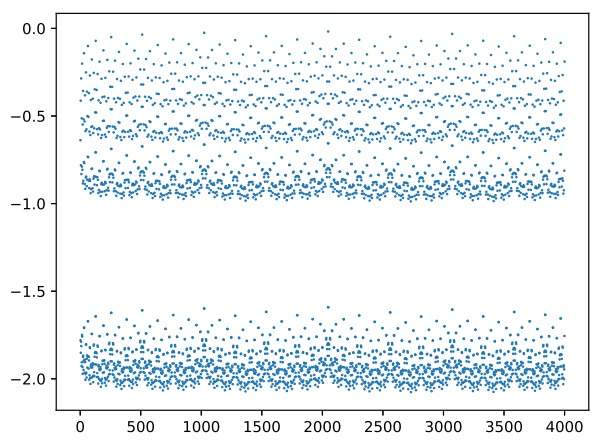

(c) $\lambda=1.5$

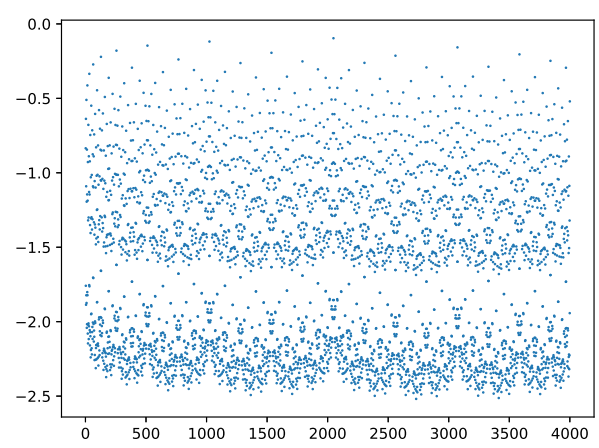

(b) $\lambda=1.3$

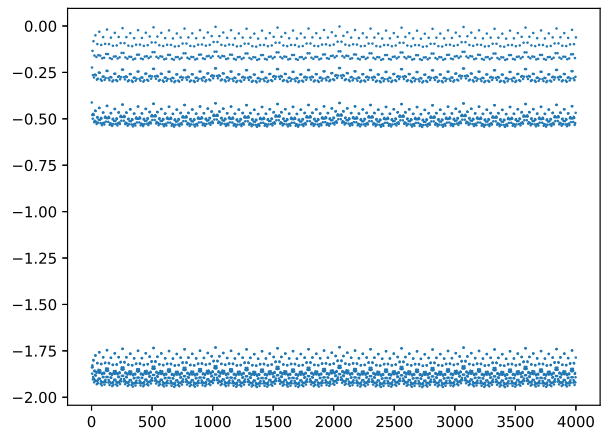

(d) $\lambda=1.7$

Figure 5: Plots of sequences (3.67) for $1<\lambda<2$ and $2 \leq N \leq 4000$.

Proof. For $N \geq 2$ as in (3.24), we have

$$
\begin{aligned}
\frac{H_{1}\left(\alpha_{N, 1}\right)-N^{2} I_{1}\left(\sigma_{1}\right)}{\log N} & =\sum_{k=1}^{p-1}\left(\sum_{j=k+1}^{p} 2^{n_{j}-n_{k}}\right) \frac{\mathcal{R}_{1}\left(2^{n_{k}+1}\right)}{\log N} \\
& +\sum_{k=1}^{p}\left(1-\sum_{j=k+1}^{p} 2^{n_{j}-n_{k}+1}\right) \frac{\mathcal{R}_{1}\left(2^{n_{k}}\right)}{\log N} .
\end{aligned}
$$

If $C$ is an upper bound for all $\left|\mathcal{R}_{1}(N)\right|$, applying (3.48) and the triangle inequality we get

$$
\left|\frac{H_{1}\left(\alpha_{N, 1}\right)-N^{2} I_{1}\left(\sigma_{1}\right)}{\log N}\right| \leq \frac{4 p C}{\log N} .
$$

It follows from (3.24) that $p \leq n_{1}+1$, and we have $\log N \geq \log \left(2^{n_{1}}\right)=n_{1} \log 2$. These inequalities and (3.80) imply the boundedness of (3.76).

We have $H_{1}\left(\alpha_{N, 1}\right)-N^{2} I_{1}\left(\sigma_{1}\right) \leq \mathcal{R}_{1}(N)$ with equality if $N=2^{n}, n \geq 1$. Since we have $\lim _{N \rightarrow \infty} \mathcal{R}_{1}(N) / \log N=0$, we obtain (3.77). 
The function $\zeta(s)$ satisfies $\zeta(-1)=-1 / 12$, hence according to (3.42) we have

$$
\lim _{N \rightarrow \infty} \mathcal{R}_{1}(N)=-\frac{\pi}{3} .
$$

Consider the subsequence $N(p):=\frac{4^{p}-1}{3}$ defined in (3.74). In virtue of (3.75), we have

$$
\frac{H_{1}\left(\alpha_{N(p), 1}\right)-N(p)^{2} I_{1}\left(\sigma_{1}\right)}{\log (N(p))}=\rho_{p, 1}+\rho_{p, 2}
$$

where

$$
\begin{aligned}
\rho_{p, 1} & :=\frac{1}{3} \frac{1}{\log (N(p))} \sum_{m=1}^{p-1}\left(1-\frac{1}{4^{m}}\right) \mathcal{R}_{1}\left(2^{2 m+1}\right), \\
\rho_{p, 2} & :=\frac{1}{3} \frac{1}{\log (N(p))} \sum_{m=0}^{p-1}\left(1+\frac{2}{4^{m}}\right) \mathcal{R}_{1}\left(2^{2 m}\right) .
\end{aligned}
$$

Let $\epsilon>0$ be fixed. By (3.81), there exists $N_{\epsilon} \in \mathbb{N}$, which we assume to be odd, such that

$$
\left|\mathcal{R}_{1}\left(2^{k}\right)+\frac{\pi}{3}\right|<\epsilon \quad \text { for all } k \geq N_{\epsilon} .
$$

Let $m_{\epsilon}:=\left(N_{\epsilon}-1\right) / 2$. Then, we can write

$$
\begin{aligned}
\rho_{p, 1} & =\frac{1}{3} \frac{1}{\log (N(p))}\left(\sum_{m=1}^{m_{\epsilon}-1}\left(1-\frac{1}{4^{m}}\right) \mathcal{R}_{1}\left(2^{2 m+1}\right)+\sum_{m=m_{\epsilon}}^{p-1}\left(1-\frac{1}{4^{m}}\right)\left(\mathcal{R}_{1}\left(2^{2 m+1}\right)+\frac{\pi}{3}\right)\right) \\
& -\frac{\pi}{9} \frac{1}{\log (N(p))} \sum_{m=m_{\epsilon}}^{p-1}\left(1-\frac{1}{4^{m}}\right) .
\end{aligned}
$$

Since $m_{\epsilon}$ is fixed, we have

$$
\lim _{p \rightarrow \infty} \frac{1}{\log (N(p))} \sum_{m=1}^{m_{\epsilon}-1}\left(1-\frac{1}{4^{m}}\right) \mathcal{R}_{1}\left(2^{2 m+1}\right)=0 .
$$

Hence, applying (3.82) we get

$$
\left|\rho_{p, 1}+\frac{\pi}{9} \frac{1}{\log (N(p))} \sum_{m=m_{\epsilon}}^{p-1}\left(1-\frac{1}{4^{m}}\right)\right| \leq o(1)+\frac{1}{3} \frac{\epsilon}{\log (N(p))} \sum_{m=m_{\epsilon}}^{p-1}\left(1-\frac{1}{4^{m}}\right) .
$$

A simple calculation gives

$$
\lim _{p \rightarrow \infty} \frac{1}{\log (N(p))} \sum_{m=m_{\epsilon}}^{p-1}\left(1-\frac{1}{4^{m}}\right)=\frac{1}{\log 4} .
$$

So, letting $p \rightarrow \infty$ and then $\epsilon \rightarrow 0$ in (3.83), we get

$$
\lim _{p \rightarrow \infty} \rho_{p, 1}=-\frac{\pi}{9 \log 4} .
$$

The same limit is valid for $\rho_{p, 2}$, hence

$$
\liminf _{N \rightarrow \infty} \frac{H_{1}\left(\alpha_{N, 1}\right)-N^{2} I_{1}\left(\sigma_{1}\right)}{\log N} \leq \lim _{p \rightarrow \infty} \frac{H_{1}\left(\alpha_{N(p), 1}\right)-N(p)^{2} I_{1}\left(\sigma_{1}\right)}{\log (N(p))}=-\frac{\pi}{9 \log 2},
$$

which justifies (3.78). 
Figure 6 shows a plot of the sequence (3.76). We remark that the value $-\pi /(9 \log 2)$ in the right-hand side of (3.78) is the lowest limit value that we can get among subsequences $\left(N_{r}(p)\right)_{p=1}^{\infty}$, where

$$
N_{r}(p)=\sum_{k=0}^{p-1} 2^{r k}=\frac{2^{r p}-1}{2^{r}-1}, \quad r \in \mathbb{N},
$$

of which the sequence $(N(p))_{p=1}^{\infty}$ considered in the proof of Theorem 3.18 is a particular case. Indeed, the reader can check that for the sequence (3.84) we have

$$
\lim _{p \rightarrow \infty} \frac{H_{1}\left(\alpha_{N_{r}(p), 1}\right)-N_{r}(p)^{2} I_{1}\left(\sigma_{1}\right)}{\log \left(N_{r}(p)\right)}=-\frac{2^{r}-2}{r\left(2^{r}-1\right)} \frac{\pi}{3 \log 2}
$$

and the largest value of $\left\{\frac{2^{r}-2}{r\left(2^{r}-1\right)}\right\}_{r \in \mathbb{N}}$ is $1 / 3$.

If $\tau(N)$ is the number of ones in the binary representation of $N$, and $\mathcal{N} \subset \mathbb{N}$ is a subsequence such that $(\tau(N))_{N \in \mathcal{N}}$ is bounded, then it follows from (3.79) that $\left(H_{1}\left(\alpha_{N, 1}\right)-N^{2} I_{1}\left(\sigma_{1}\right)\right)_{N \in \mathcal{N}}$ is bounded, and therefore

$$
\lim _{N \in \mathcal{N}} \frac{H_{1}\left(\alpha_{N, 1}\right)-N^{2} I_{1}\left(\sigma_{1}\right)}{\log N}=0 .
$$

So in order to obtain non-zero limit points of the sequence (3.76), such as the limits (3.85), one needs to take subsequences $\mathcal{N}$ such that $\lim _{N \in \mathcal{N}} \tau(N)=\infty$.

\section{The case $\lambda=2$}

Theorem 4.1. Let $d \geq 1$ be arbitrary. A sequence $\left(a_{n}\right)_{n=0}^{\infty} \subset S^{d}$ is a greedy $\lambda$-energy sequence for $\lambda=2$, if and only if

$$
a_{2 k+1}=-a_{2 k}, \quad \text { for every } \quad k \geq 0 .
$$

If $\left(a_{n}\right)_{n=0}^{\infty} \subset S^{d}$ is such a sequence, then for each $n \geq 1$,

$$
\begin{aligned}
H_{2}\left(\alpha_{2 n, 2}\right) & =8 n^{2} \\
H_{2}\left(\alpha_{2 n+1,2}\right) & =8\left(n^{2}+n\right) .
\end{aligned}
$$

For the potential (1.7) we have, for each $n \geq 1$,

$$
U_{2 n-1}\left(a_{2 n-1}\right)=U_{2 n}\left(a_{2 n}\right)=4 n .
$$

In particular,

$$
\lim _{N \rightarrow \infty} \frac{H_{2}\left(\alpha_{N, 2}\right)}{N^{2}}=\lim _{n \rightarrow \infty} \frac{U_{n}\left(a_{n}\right)}{n}=2 .
$$

Proof. The proof is based on the fact that for any $a, x \in S^{d}$,

$$
|x-a|^{2}+|x+a|^{2}=4 .
$$

Let $\left(a_{n}\right)_{n=0}^{\infty} \subset S^{d}$ be a greedy $\lambda$-energy sequence for $\lambda=2$. Clearly, (4.1) holds for $k=0$. Assume that (4.1) is valid for all $0 \leq k \leq n$. By definition, the point $a_{2 n+3}$ must maximize the function

$$
\begin{aligned}
\sum_{k=0}^{n}\left(\left|x-a_{2 k}\right|^{2}+\left|x-a_{2 k+1}\right|^{2}\right)+\left|x-a_{2 n+2}\right|^{2} & =\sum_{k=0}^{n}\left(\left|x-a_{2 k}\right|^{2}+\left|x+a_{2 k}\right|^{2}\right)+\left|x-a_{2 n+2}\right|^{2} \\
& =4(n+1)+\left|x-a_{2 n+2}\right|^{2}, \quad x \in S^{d} .
\end{aligned}
$$




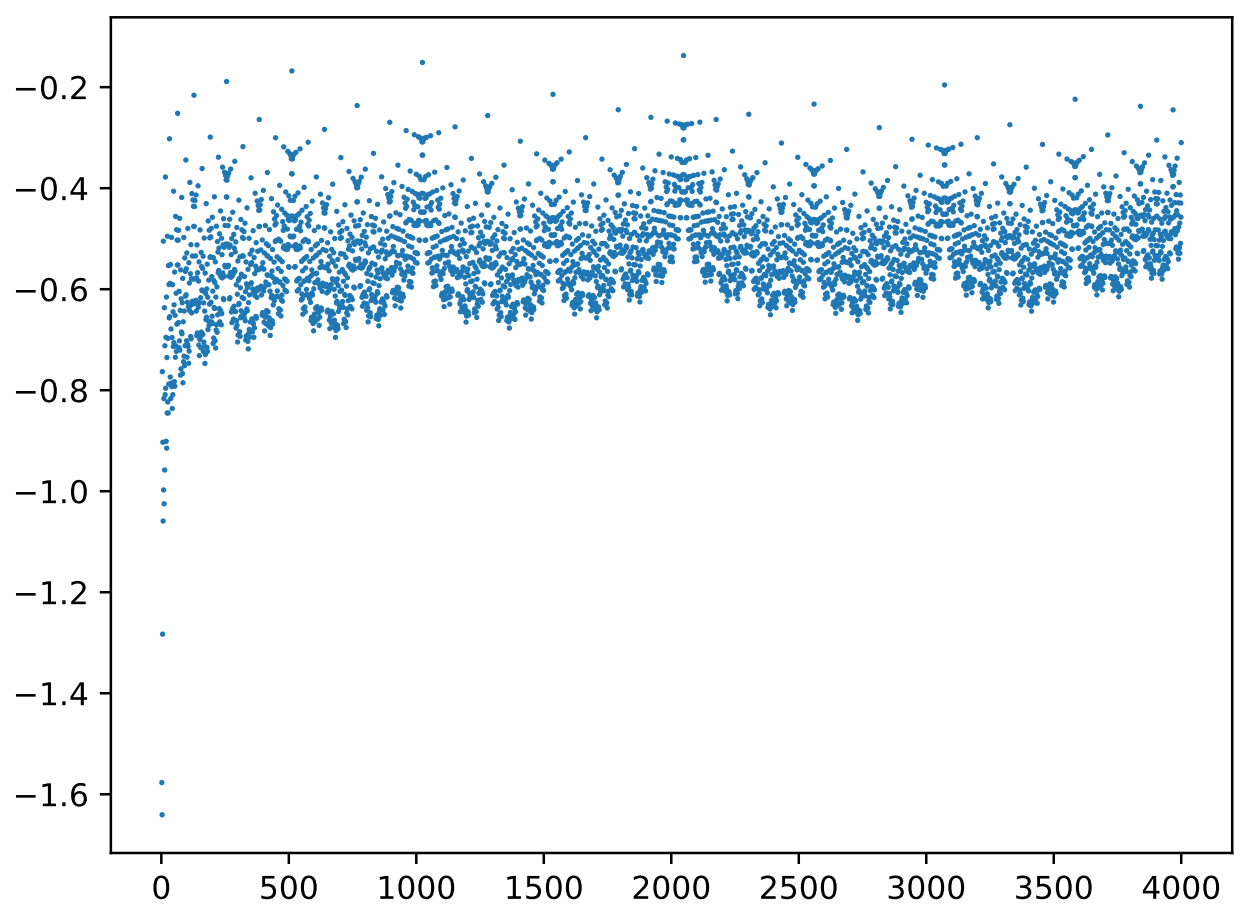

Figure 6: Plot of the sequence (3.76) for $2 \leq N \leq 4000$, in the case $\lambda=1$.

Hence $a_{2 n+3}=-a_{2 n+2}$, which proves (4.1) for $k=n+1$. Conversely, it is clear that any sequence $\left(a_{n}\right)_{n=0}^{\infty} \subset S^{d}$ that satisfies (4.1), is a greedy $\lambda$-energy sequence for $\lambda=2$.

One proves (4.2) by induction. This identity is valid for $n=1$. Assume that it is valid for $n$. Since

$$
H_{2}\left(\alpha_{2 n+2}\right)=H_{2}\left(\alpha_{2 n+1}\right)+2 \sum_{j=0}^{2 n}\left|a_{2 n+1}-a_{j}\right|^{2}
$$

pairing $\left\{a_{2 k}, a_{2 k+1}\right\}=\left\{a_{2 k},-a_{2 k}\right\}$ in the summation expression and using (4.3), we obtain $H_{2}\left(\alpha_{2 n+2}\right)=8(n+1)^{2}$. Similarly, one checks that $H_{2}\left(\alpha_{2 n+3}\right)=8(n+2)(n+1)$.

The rest of the claims follow immediately.

Remark 4.2. From (4.1) and (4.3) we deduce that the function $U_{2 k}(x) \equiv 4 k$ is constant on $S^{d}$, for every $k \geq 1$.

Remark 4.3. Our reviewer has indicated to us the interesting formula

$$
U_{n}(x)=2 n-2\left\langle x, \sum_{k=0}^{n-1} a_{k}\right\rangle, \quad x \in S^{d},
$$

valid for $\lambda=2$. This formula can be used for an alternative proof of Theorem 4.1 . 
Remark 4.4. If $\left(a_{n}\right)_{n=0}^{\infty} \subset S^{d}$ is a greedy $\lambda$-energy sequence for $\lambda=2$, then the associated sequence $\left(\sigma_{N}\right)$ defined in (1.5) is not necessarily convergent. The following sequence on $S^{2} \subset \mathbb{R}^{3}$ illustrates this claim. Let

$$
y_{0}:=(1,0,0), \quad y_{1}:=(-1,0,0), \quad y_{2}:=(0,1,0), \quad y_{3}:=(0,-1,0),
$$

and let the first four points of the sequence be defined as

$$
a_{i}:=y_{i}, \quad 0 \leq i \leq 3
$$

The rest of the sequence is constructed inductively. Suppose that for an integer $m \geq 2$, the configuration $\alpha_{2^{m}}$ formed by the first $2^{m}$ points of the sequence, consists of $2^{m-2}$ points at each $y_{i}, 0 \leq i \leq 3$. Then, we have

$$
\sigma_{2^{m}}=\frac{2^{m-2}}{2^{m}}\left(\delta_{y_{0}}+\delta_{y_{1}}+\delta_{y_{2}}+\delta_{y_{3}}\right)=\frac{1}{4}\left(\delta_{y_{0}}+\delta_{y_{1}}+\delta_{y_{2}}+\delta_{y_{3}}\right) .
$$

We take the next $2^{m-1}$ points alternating between $y_{0}$ and $y_{1}=-y_{0}$, i.e., we define

$$
a_{2^{m}+k-1}:=(-1)^{k-1} y_{0}, \quad 1 \leq k \leq 2^{m-1} .
$$

Then, the configuration $\alpha_{2^{m}+2^{m-1}}=\alpha_{3 \cdot 2^{m-1}}$ consists of $2^{m-1}$ points at each $y_{0}, y_{1}$, and $2^{m-2}$ points at each $y_{2}, y_{3}$. Thus,

$$
\sigma_{3 \cdot 2^{m-1}}=\frac{1}{3}\left(\delta_{0}+\delta_{1}\right)+\frac{1}{6}\left(\delta_{y_{2}}+\delta_{y_{3}}\right) .
$$

Now we take the next $2^{m-1}$ points alternating between $y_{2}$ and $y_{3}=-y_{2}$, i.e., we define

$$
a_{3 \cdot 2^{m-1}+k-1}:=(-1)^{k-1} y_{2}, \quad 1 \leq k \leq 2^{m-1} .
$$

With this definition, we have constructed the configuration $\alpha_{2^{m+1}}$, consisting of $2^{m-1}$ points at each $y_{i}, 0 \leq i \leq 3$. Therefore,

$$
\sigma_{2^{m+1}}=\sigma_{2^{m}}
$$

and the inductive construction is concluded. Since (4.5) and (4.6) are valid for every $m \geq 2$, we see that the sequence $\left(\sigma_{N}\right)$ is not convergent.

However, (4.1) implies that any convergent subsequence of $\left(\sigma_{N}\right)$ will converge to a probability measure $\sigma$ on $S^{d}$ with center of mass at the origin, i.e., $\sigma$ satisfies

$$
\int_{S^{d}} x_{i} d \sigma(x)=0, \quad 1 \leq i \leq d+1, \quad x=\left(x_{1}, \ldots, x_{d+1}\right) .
$$

\section{The case $\lambda>2$}

Lemma 5.1. Let $d \geq 1$, and let $a \in S^{d}$ be fixed. If $\lambda>2$, the function

$$
|x-a|^{\lambda}+|x+a|^{\lambda}, \quad x \in S^{d},
$$

attains its maximum value only at the points $x=a$ and $x=-a$. 
Proof. In the case $d=1$, the claim is equivalent to show that the function

$$
f(\theta):=\left|e^{i \theta}-1\right|^{\lambda}+\left|e^{i \theta}+1\right|^{\lambda}, \quad 0 \leq \theta \leq \pi,
$$

attains its maximum value on the interval $[0, \pi]$ only at the points $\theta=0$ and $\theta=\pi$. Indeed,

$$
\begin{aligned}
f(\theta) & =2^{\lambda}\left(\sin ^{\lambda}\left(\frac{\theta}{2}\right)+\cos ^{\lambda}\left(\frac{\theta}{2}\right)\right) \\
f^{\prime}(\theta) & =\lambda 2^{\lambda-1}\left(\sin ^{\lambda-1}\left(\frac{\theta}{2}\right) \cos \left(\frac{\theta}{2}\right)-\cos ^{\lambda-1}\left(\frac{\theta}{2}\right) \sin \left(\frac{\theta}{2}\right)\right), \quad 0<\theta<\pi .
\end{aligned}
$$

Hence, the only critical point of $f$ on $(0, \pi)$ is $\theta=\pi / 2$. We have $f(0)=f(\pi)=2^{\lambda}>f(\pi / 2)=$ $2^{1+\frac{\lambda}{2}}$, which proves the claim in the case $d=1$.

The proof can be completed now using induction on $d$. Assume the result holds on $S^{d-1}$, for some $d \geq 2$. Let $a \in S^{d}$ be fixed, and let $x \in S^{d} \backslash\{a,-a\}$. Since $d \geq 2$, there exists a hyperplane $\pi$ of $\mathbb{R}^{d+1}$ that passes through the origin and contains the points $a,-a, x$. The set $S^{d} \cap \pi$, which contains these three points, is isometric to $S^{d-1}$, which can be identified, for example, with the subset of $S^{d}$ given by $S^{d} \cap\left[x_{d+1}=0\right]$. By the induction hypothesis, $|x-a|^{\lambda}+|x+a|^{\lambda}<2^{\lambda}$. Since $x \in S^{d} \backslash\{a,-a\}$ was taken arbitrarily, the result is proven.

Theorem 5.2. Let $d \geq 1$ be arbitrary, and let $\left(a_{n}\right)_{n=0}^{\infty} \subset S^{d}$ be a greedy $\lambda$-energy sequence for $\lambda>2$. Then, for each $k \geq 0$,

$$
\left\{a_{2 k}, a_{2 k+1}\right\}=\left\{a_{0},-a_{0}\right\} .
$$

For each $n \geq 1$,

$$
\begin{aligned}
H_{\lambda}\left(\alpha_{2 n, \lambda}\right) & =2^{\lambda+1} n^{2} \\
H_{\lambda}\left(\alpha_{2 n+1, \lambda}\right) & =2^{\lambda+1}\left(n^{2}+n\right) .
\end{aligned}
$$

For the potential (1.7) we have, for each $n \geq 1$,

$$
U_{2 n-1}\left(a_{2 n-1}\right)=U_{2 n}\left(a_{2 n}\right)=n 2^{\lambda} .
$$

In particular,

$$
\lim _{N \rightarrow \infty} \frac{H_{\lambda}\left(\alpha_{N, \lambda}\right)}{N^{2}}=\lim _{n \rightarrow \infty} \frac{U_{n}\left(a_{n}\right)}{n}=2^{\lambda-1},
$$

and

$$
\sigma_{N, \lambda} \stackrel{*}{\longrightarrow} \frac{1}{2} \delta_{a_{0}}+\frac{1}{2} \delta_{-a_{0}} .
$$

Proof. Let $\left(a_{n}\right)_{n=0}^{\infty} \subset S^{d}$ be a greedy $\lambda$-energy sequence for $\lambda>2$. Obviously, $a_{1}=-a_{0}$, so (5.1) holds for $k=0$. Assume that (5.1) is valid for all $0 \leq k \leq n-1$, for some $n \geq 1$. Then $\alpha_{2 n, \lambda}$ consists of $n$ points at $x=a_{0}$ and $n$ points at $x=-a_{0}$. So the point $a_{2 n}$ must maximize the function

$$
\sum_{k=0}^{2 n-1}\left|x-a_{k}\right|^{\lambda}=n\left(\left|x-a_{0}\right|^{\lambda}+\left|x+a_{0}\right|^{\lambda}\right), \quad x \in S^{d} .
$$

By Lemma 5.1, we have $a_{2 n}=a_{0}$ or $a_{2 n}=-a_{0}$. If $a_{2 n}=a_{0}$, then $a_{2 n+1}$ must maximize the function

$$
n\left(\left|x-a_{0}\right|^{\lambda}+\left|x+a_{0}\right|^{\lambda}\right)+\left|x-a_{0}\right|^{\lambda},
$$

which clearly has a unique maximum at $x=-a_{0}$, so $a_{2 n+1}=-a_{0}$. Similarly, $a_{2 n}=-a_{0}$ implies that $a_{2 n+1}=a_{0}$. So (5.1) is proved by induction.

The rest of the claims follow immediately. 
Remark 5.3. For $\lambda>2$, the energy of any maximal distribution is $2^{\lambda-1}$. It follows from Theorem 5.2 that for $\lambda>2$ and $N \geq 2$,

$$
H_{\lambda}\left(\alpha_{N, \lambda}\right)-N^{2} 2^{\lambda-1}= \begin{cases}0 & \text { if } N \text { is even } \\ -2^{\lambda-1} & \text { if } N \text { is odd }\end{cases}
$$

For $n \geq 1$

$$
U_{n}\left(a_{n}\right)-n 2^{\lambda-1}= \begin{cases}0 & \text { if } n \text { is even } \\ 2^{\lambda-1} & \text { if } n \text { is odd } .\end{cases}
$$

These identities are also valid for $\lambda=2$.

Acknowledgements: We thank the reviewer of this paper for sharing with us an interesting approach to the study of some of the problems discussed in this work.

\section{References}

[1] L. Białas-Cież and J.-P. Calvi, Pseudo Leja sequences, Ann. Mat. Pura Appl. 191 (2012), 53-75.

[2] G. Björck, Distributions of positive mass, which maximize a certain generalized energy integral, Ark. Mat. 3 (1956), 255-269.

[3] S.V. Borodachov, D.P. Hardin, and E.B. Saff, Discrete Energy on Rectifiable Sets, Springer Monographs in Mathematics, 2019, Springer.

[4] J.S. Brauchart, D.P. Hardin, and E.B. Saff, The Riesz energy of the N-th roots of unity: an asymptotic expansion for large N, Bull. Lond. Math. Soc. 41 (2009), no. 4, 621-633.

[5] D. Coroian and P. Dragnev, Constrained Leja points and the numerical solution of the constrained energy problem, J. Comput. Appl. Math. 131 (2001), 427-444.

[6] A. Edrei, Sur les déterminants récurrents et les singularités d'une fonction donnée par son développement de Taylor, Compositio Mathematica 7 (1939), 20-88.

[7] J. Górski, Les suites de points extrémaux liés aux ensembles dans l'espace à 3 dimensions, Ann. Polon. Math. 4 (1957), 14-20.

[8] M. Götz, On the distribution of Leja-Górski points, J. Comput. Anal. Appl. 3 (2001), 223241.

[9] D.P. Hardin, A. Reznikov, E.B. Saff, and A. Volberg, Local properties of Riesz minimal energy configurations and equilibrium measures, Int. Math. Res. Not. IMRN 2019, no. 16, 5066-5086.

[10] F. Leja, Sur certaines suites liées aux ensembles plans et leur application à la représentation conforme, Ann. Polon. Math. 4 (1957), 8-13.

[11] A. López García, Greedy energy points with external fields, in "Recent Trends in Orthogonal Polynomials and Approximation Theory" (J. Arvesú, F. Marcellán, and A. MartínezFinkelshtein, eds.), Contemp. Math. 507, 2010, 189-207. 
[12] A. López García and E.B. Saff, Asymptotics of greedy energy points, Math. Comp. 79 (2010), 2287-2316.

[13] A. López-García and D.A. Wagner, Asymptotics of the energy of sections of greedy energy sequences on the unit circle, and some conjectures for general sequences, Comput. Methods Funct. Theory 15 (2015), 721-750.

[14] I.E. Pritsker, Equidistribution of points via energy, Ark. Mat. 49 (2011), 149-173.

[15] I.E. Pritsker, Distribution of point charges with small discrete energy, Proc. Amer. Math. Soc. 139 (2011), 3461-3473.

[16] E.B. Saff and V. Totik, Logarithmic Potentials with External Fields, Grundlehren der mathematischen Wissenschaften 316, Springer, 1997.

[17] J. Siciak, Two criteria for the continuity of the equilibrium Riesz potentials, Comment. Math. Prace Mat. 14 (1970), 91-99.

[18] B. Simanek, Asymptotically optimal configurations for Chebyshev constants with an integrable kernel, New York J. Math. 22 (2016), 667-675.

[19] K.B. Stolarsky, The sum of the distances to certain pointsets on the unit circle, Pac. J. of Math. 59 (1975), 241-251. 